\title{
High Aspect Ratio Wing Design: Optimal Aerostructural Tradeoffs for the Next Generation of Materials
}

\author{
Graeme J. Kennedy* \\ Georgia Institute of Technology, School of Aerospace Engineering, Atlanta, Georgia \\ Gaetan W. Kenway ${ }^{\dagger}$ and Joaquim R. R. A. Martins ${ }^{\ddagger}$ \\ University of Michigan, Department of Aerospace Engineering, Ann Arbor, Michigan
}

\begin{abstract}
Current and future composite material technologies have the potential to greatly improve the performance of large transport aircraft. However, the coupling between aerodynamics and structures makes it challenging to design optimal flexible wings, and the transonic flight regime requires high fidelity computational models. We address these challenges by solving a series of medium- and highfidelity aerostructural optimization problems that explore the design space for the wing of a large transport aircraft. We consider three different materials: aluminum, carbon-fiber reinforced composites and an hypothetical composite based on carbon nanotubes. The design variables consist of both aerodynamic shape (including span), and structural sizing, as well as ply angle fractions in the case of composites. Pareto fronts with respect to takeoff weight and fuel burn are generated. The wing performance in each case is optimized subject to stress and buckling constraints. We found that composite wings consistently resulted in lower fuel burn and lower structural weight, and that the carbon nanotube composite did not yield the increase in performance one would expect from a material with such outstanding properties. This was in part due to the minimum structural thickness constraint. For all materials, the minimum fuel burn wings were found to be longer, heavier, thinner, more flexible, and more lightly loaded than their minimum TOGW counterparts.
\end{abstract}

\section{Introduction}

The growing concern about the effect of green-house gas emissions on climate change, together with the rise in fuel prices, has lead to increased research on efficient aircraft. This research is crucial if aviation is to remain sustainable, both from the environmental and from the economic viewpoints. To this end, NASA has been developing computational tools, technologies, and concepts to make significant improvements in the energy efficiency of commercial aviation.

NASA's high aspect ratio wing work under the Fixed Wing project, in particular, aims to explore ways to increase wing aspect ratio in order to reduce fuel burn [9]. The minimization of fuel burn represents a tradeoff between structural weight minimization and drag minimization. However, the fuel burn minimization tradeoff is biased towards designs that have higher structural weights than current aircraft, as current aircraft are designed to strike a balance between acquisition cost (which correlates with weight) and operation cost (which depends mostly on fuel burn).

As a consequence of seeking better fuel burn due to higher fuel prices, the trend in the wing design of transport aircraft has been to increase the aspect ratio to improve the lift-to-drag ratio $(L / D)$. The Boeing 787, for example, has an aspect ratio of 11 , which is $10 \%$ higher than the Boeing 777-300ER $(A R=10)$ and even higher than the older Boeing 747-400 $(A R=8)$. This trend is driven by increases in fuel price, which push the design tradeoffs toward higher $L / D$. This increase in aspect ratio has also been observed in previous aerostructural design optimization studies by the authors [14, 12]. This was such an important factor for the planned Boeing $777 \mathrm{X}$ that it will use a folding mechanism (which incurs an additional weight penalty) to enable a large span while remaining in the same gate span constraint code.

To enable larger wing aspect ratios we can: (1) develop and implement new airframe technologies and (2) develop and apply new design methodologies that maximize the benefit of a new airframe technology. In this work, we focus on the latter by considering new materials and exploring the wing design space using medium- and high-fidelity multidisciplinary design optimization (MDO) methods. This wing design exploration requires the consideration of both aerodynamics and structures, not only due to the tradeoffs between drag and structural weight we just mentioned,

\footnotetext{
*Assistant Professor, AIAA Member

${ }^{\dagger}$ Postdoctoral Research Fellow, AIAA Member

${ }^{\ddagger}$ Associate Professor, AIAA Associate Fellow
} 
but also due to the wing flexibility, which intrinsically couples these two disciplines. This is especially important in the high aspect ratio wings considered in this study, since wings become more flexible as aspect ratio is increased. To consider this coupling, we use aerostructural optimization, which simultaneously performs the structural sizing and the aerodynamic shape optimization, naturally leading to optimally static aeroelastic tailored wings. Such wings exhibit high aerodynamic performance at cruise conditions while avoiding an excessive weight penalty (which is determined by structural sizing at critical flight conditions). This is made possible largely by tailoring the wing flexibility to achieve passive load alleviation, as shown previously by the authors [14].

Since the bulk of commercial air transport is performed by aircraft operating in the transonic flow regime, it is imperative to use computational fluid dynamics (CFD) aerodynamic models that consider compressibility effects. On the structural side, it is important to include a detailed model of the structural wing box, since an accurate quantification of the tradeoff between aerodynamic shape and wing weight is required.

Although there has been much research into aerodynamic shape optimization based on the solution of the Euler equations, we have found that the resulting airfoil shapes differ significantly from shapes obtained with optimization based on the Reynolds-averaged Navier-Stokes (RANS) equations [19]. Previous results in Euler-based aerostructural optimization also show that unrealistic airfoil shapes are obtained due to the inability of the Euler equations to predict the shock strength and position, which is strongly coupled to viscous effects [14]. Since the cost of RANS-based optimization is high, the approach we take in the present work is to use a much faster panel method for a broader exploration of the aerostructural design space (as previously done by Kennedy and Martins [12]), and then select smaller design spaces of interest for further refinement using RANS-based aerostructural optimization.

The quantification of the tradeoff between acquisition cost and operation cost varies significantly between airlines, aircraft types, and is highly dependent on fuel price. Therefore, it is important to consider various objective functions between the two extremes of minimum fuel burn and minimum weight. To address this issue, we study optimal designs that minimize fuel burn, takeoff gross weight (TOGW), and several objectives representing a compromise between these two. The result of this multiobjective study is a Pareto front that quantifies the weight penalty of minimizing fuel burn relative to the minimum TOGW design, as well as how that weight penalty varies as the objective varies between fuel burn and TOGW.

When it comes to the technology, we chose to explore the use of different materials and seek the answers to two questions: (1) Can we take further advantage of carbon-fiber reinforced polymer (CFRP) composites by tailoring of the flexibility through the optimization of ply angle orientation, and (2) What if we had a material that had an order of magnitude higher strength and stiffness relative to CFRP? How well would a wing made with such material perform, and what would it look like?

To answer these questions, we consider three different materials: an aluminum alloy, a CFRP composite, and an hypothetical composite based on carbon nanotube (CNT) fibers. Aluminum alloys have been used for many decades and are well understood, so aluminum is used to provide a reference. While CFRP composites are now used in the primary structure of the two latest wide-body airliners (the Boeing 787 and Airbus A350), the design of CFRP composite wings still does not take full advantage of the additional design freedom provided by ply angle orientations. Finally, the CNT-based composite results should give us an idea of what wing designs will look like when vastly superior materials are available.

In the following sections, we describe a series of structural and aerostructural design problems that we solved. The objective of this sequence of design problems is to evaluate the impact of new structural material technologies on the tradeoffs in the design of a conventional aircraft wing. In order to perform this assessment, we use a series of structural and coupled aerostructural optimization problems. Optimization of each candidate design is necessary to make a fair comparison between the different materials, so that the full benefit of the potential technology is realized. Comparing sub-optimal designs would lead to incorrect conclusions regarding the relative merits of different materials. However, the design optimization problem must also adequately capture the requirements and objective of a realistic aircraft design problem, otherwise the predicted performance improvements may be a result of an incomplete analysis or an inadequate design.

The remainder of this paper is structured as follows: in Section II we describe the essential aspects of the analysis and design tools used in this study. The parametric baseline wing that was used as the starting point for all the optimizations is described in Section III. In Section IV we describe the details of the structural design parametrizations that we used, followed by the aerostructural design problem formulation in Section V. The results are presented and discussed in Section VI, and then we finish with conclusions and recommendations for future work in the last section. 


\section{Analysis and design tools}

As previously mentioned, it is crucial to consider the coupling between the structural flexibility and aerodynamic loads when performing wing analysis and design. In this study, this is achieved through static aeroelastic analysis, or aerostructural analysis, where the aerodynamic forces on the deformed flying shape of the aircraft are in equilibrium with the internal forces. All the functions of interest in the design problem we consider-objective functions and constraints-are evaluated using aerostructural analysis to capture the effect of the structural flexibility on cruise aerodynamic performance, as well as the effect of the aerodynamic shape on the structural stresses. This is particularly important for higher aspect ratio wings, which tend to be more flexible.

To perform aerostructural analysis, we use both a medium fidelity method that uses a panel method (with compressibility, viscous, and wave drag corrections) coupled to a high-fidelity finite-element solver, as well as a RANS CFD solver coupled to the same finite-element solver. In this section we provide a brief description of these aerodynamic and structural solver, and how they are coupled to perform aerostructural analysis. More details are provided by Kennedy and Martins [11] and Kenway et al. [15].

Due to the large dimensionality of the design space and the computational cost of the analysis, we use a gradientbased optimization algorithm $[4,27]$ together with an adjoint method for evaluating the required gradients. Adjoint methods enable the efficient evaluation of gradients in large dimensional spaces and have been used in both aerodynamic shape $[7,20,18]$ and structural design optimization $[34,33,28]$. Since we are considering aerostructural coupling, we require a coupled adjoint that computes gradients of the aerostructural system with respect to both external shape and internal structural sizing. Again, we provide only a brief description of these techniques in this section; additional details can be found in our previous work $[11,15]$.

\section{A. Aerodynamic analysis}

The medium-fidelity aerodynamic analysis consists of a panel code that computes the pressure distribution over the wing, which is then transfered to the structural analysis. Since this panel code is only able to compute induced drag, we also estimate the viscous drag and the drag due to compressibility. The RANS CFD includes all these drag components.

\section{Panel code and induced drag}

For the medium-fidelity aerostructural cases, the aerodynamic analysis is performed using TriPan, an unstructured, three-dimensional parallel panel code for calculating the aerodynamic forces, moments, and pressures for inviscid, incompressible, external lifting flows using the Prandtl-Glauert equation [11]. TriPan uses constant first-order source and doublet singularity elements distributed over the entire lifting surface and doublet elements distributed over the wake [10]. The source strengths are determined based on the onset flow conditions, while the boundary conditions for the doublet strengths form a dense linear system of equations, represented here by

$$
\mathbf{R}_{A}(\mathbf{w}, \mathbf{u})=0,
$$

where $\mathbf{u}$ and $\mathbf{w}$ are vectors of the structural and aerodynamic state variables, respectively. The linear system represented by Equation (1) is solved in parallel using PETSc [1]. A dense matrix format is used for the matrix-vector products, while a sparse approximate-Jacobian is used to form a incomplete LU (ILU) preconditioner. The linear system is solved using the Krylov subspace method GMRES.

The lift-induced drag is computed directly from TriPan using a Trefftz-plane integration.

\section{Viscous drag}

The viscous drag is computed by dividing the wing into chordwise strips, estimating the profile drag coefficients of the corresponding airfoil sections, and then integrating the drag in the spanwise direction. The profile drag coefficients are computed based on a quadratic model:

$$
c_{d p}=c_{d 0}+c_{d 2} c_{l}^{2},
$$

where $c_{l}$ is the sectional lift coefficient, and $c_{d 0}$ and $c_{d 2}$ are coefficients defined below. The coefficient $c_{d 0}$ is based on the skin friction estimate

$$
c_{d 0}=F_{c} c_{f},
$$


where $c_{f}$ is the turbulent skin-friction coefficient determined using the van Driest II method [6], and $F_{c}$ is a factor that corrects this estimate to account for form drag. This factor is given by an empirical function of the airfoil thicknessto-chord ratio, $t / c$, as follows:

$$
F_{c}=1+2.7\left(\frac{t}{c}\right)+100\left(\frac{t}{c}\right)^{4} .
$$

Finally, the quadratic coefficient in Equation (2) is obtained using the empirical relationship by Wakayama and Kroo [35]:

$$
c_{d 2}=\frac{0.38}{\cos ^{2} \Lambda} c_{d 0},
$$

where $\Lambda$ is the leading-edge sweep angle.

\section{Compressibility drag}

The compressibility drag is computed based on a crest-critical Mach number computed using the Korn equation [22],

$$
M_{\text {crit }}=\frac{\kappa_{A}}{\cos \Lambda}-\frac{t / c}{\cos ^{2} \Lambda}-\frac{c_{l}}{10 \cos ^{3} \Lambda}-\left(\frac{0.1}{80}\right)^{1 / 3},
$$

where $\kappa_{A}$ is a technology factor that we set to $\kappa_{A}=0.95$, which is suitable for the supercritical airfoil sections commonly used in transport aircraft. The sectional contribution to the compressibility drag is then computed using

$$
c_{d_{c}}=20\left(M-M_{\text {crit }}\right)^{4}
$$

for $M>M_{\text {crit }}$.

\section{CFD solver}

For the high-fidelity cases, we use the CFD solver SUmb, which is a second-order structured block-based finitevolume solver for the Euler, Navier-Stokes and RANS equations [31]. SUmb uses a multi-grid solution method with an explicit Runge-Kutta time integration method with residual smoothing. An adjoint method was implemented for SUmb by Mader et al. [21] and Lyu et al. [19], enabling RANS-based aerodynamic shape optimization [18].

\section{B. Load transfer}

The load and displacement transfer scheme follows the work of Brown [2]. The displacements from the structures are extrapolated to the aerodynamic nodes using rigid links. These rigid links are formed by locating the closest point on the structural surface to each of the aerodynamic nodes. The structural surface is determined by interpolating between structural nodes using the finite-element shape functions. The displacements $\mathbf{u}_{S}$ and rotations $\boldsymbol{\theta}_{S}$ on the structural surface, and the rigid links $\mathbf{r}$ are used to determine the displacements of the aerodynamic nodes $\mathbf{u}_{A}$ as follows:

$$
\mathbf{u}_{A}=\mathbf{u}_{S}+\boldsymbol{\theta}_{S} \times \mathbf{r} .
$$

Note that this formula uses a small angle approximation. Equation (5) can be used in conjunction with the method of virtual work to form the consistent force vector for the aerodynamic forces at the structural nodes. More details of this approach are outlined by Kennedy and Martins [11].

\section{Structural analysis}

The structural analysis is performed using the Toolkit for the Analysis of Composite Structures (TACS), a parallel, finite-element code designed specifically for the analysis of stiffened, thin-walled, composite structures using either linear or geometrically nonlinear strain relationships Kennedy and Martins [11]. To date, we have exclusively used linear structural analysis. However, geometrically nonlinear analysis may be required to accurately predict the flying shape of very flexible aircraft. In addition, we have focused on using a smeared stiffness approach in which the effect of stiffeners are included in the stiffness of the skin as described in detail below. As a result, we have not used the full stiffened shell analysis capabilities of TACS. When modeling stiffened shell structures, we typically use third-order finite-element discretizations that provide a good tradeoff between solution accuracy and gradient-evaluation costs.

The residuals of the structural governing equations are

$$
\mathbf{R}_{S}(\mathbf{w}, \mathbf{u})=\mathbf{S}_{c}(\mathbf{u})-\mathbf{F}(\mathbf{w}, \mathbf{u}),
$$


where $\mathbf{u}$ is a vector of displacements and rotations, $\mathbf{S}_{c}$ are the residuals due to conservative forces and internal strain energy and $\mathbf{F}$ are the follower forces due to aerodynamic loads.

The Jacobian of the structural residuals involves two terms: the stiffness, or tangent stiffness matrix $\mathbf{K}=\partial \mathbf{S}_{c} / \partial \mathbf{u}$, and the derivative of the consistent force vector with respect to the structural displacements. This results in the following expression for the Jacobian of the structural residuals:

$$
\frac{\partial \mathbf{R}_{S}}{\partial \mathbf{u}}=\mathbf{K}-\frac{\partial \mathbf{F}}{\partial \mathbf{u}} .
$$

While the matrices involved in structural problems are typically symmetric, the term $\partial \mathbf{F} / \partial \mathbf{u}$ is non-symmetric due to the non-conservative nature of the aerodynamic forces. These non-symmetric matrices require solution algorithms different from those typically employed in structural finite-element codes. We use GMRES [29] to solve the nonsymmetric, linear systems involving the matrix in Equation (7).

\section{Approximate Newton-Krylov method}

The aerostructural residuals are the concatenation of the aerodynamic and structural residuals, represented by:

$$
\mathbf{R}(\mathbf{q}, \mathbf{x})=\left[\begin{array}{l}
\mathbf{R}_{A}(\mathbf{w}, \mathbf{u}, \mathbf{x}) \\
\mathbf{R}_{S}(\mathbf{w}, \mathbf{u}, \mathbf{x})
\end{array}\right]=0
$$

where $\mathbf{R}_{A}$ and $\mathbf{R}_{S}$ are the aerodynamic and structural residuals, $\mathbf{w}$ and $\mathbf{u}$ are the aerodynamic and structural state variables, $\mathbf{q}$ is the full set of aerostructural state variables $\mathbf{q}^{T}=\left[\mathbf{w}^{T}, \mathbf{u}^{T}\right]$, and $\mathbf{x}$ is a vector of design variables.

Newton's method applied to Equation (8) results in the following linear system of equations for the update $\Delta \mathbf{q}^{(n)}$,

$$
\frac{\partial \mathbf{R}}{\partial \mathbf{q}} \Delta \mathbf{q}^{(n)}=-\mathbf{R}\left(\mathbf{q}^{(n)}\right),
$$

where the $n^{\text {th }}$ update is applied as follows: $\mathbf{q}^{(n+1)}=\mathbf{q}^{(n)}=\Delta \mathbf{q}^{(n)}$. In our Newton-Krylov approach, we solve an approximate linearization of the system (9) inexactly, to a loose tolerance, using a Krylov subspace method. We use an approximate linearization that is less expensive to compute, which leads to better overall solution performance. We form a preconditioner for the Krylov subspace method based on discipline-level preconditioners by dropping the off-diagonal coupling terms. Note that these coupling terms are retained for the matrix-vector products, which are computed using a product-rule implementation that is discussed in further detail in Kennedy and Martins [11].

\section{E. Adjoint-based gradient computation}

Efficient gradient-based optimization requires the accurate and efficient evaluation of gradients of the objective function and constraints. In the aerostructural optimization problem we solve in this work, there are typically far fewer objective and constraint functions than there are design variables. While there are various sensitivity methods that can be used to compute accurate derivatives [23], the coupled adjoint method [26, 25], is the most computationally efficient for coupled systems when the gradients of a small number of functions of interest are required with respect to many design variables. We have developed a coupled aerostructural adjoint that is based entirely on analytic derivatives without the use of costly finite-difference approximations. Further details of this approach are presented in Kennedy and Martins [11]. The aerostructural adjoint equations can be written in the following form:

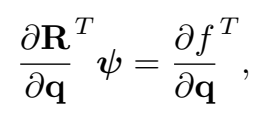

where $\boldsymbol{\psi}$ is the adjoint vector and $f(\mathbf{q}, \mathbf{x})$ is either an aerodynamic or structural function of interest. Once the adjoint vector is determined using Equation (10), the total derivative is computed using:

$$
\nabla_{\mathbf{x}} f=\frac{\partial f}{\partial \mathbf{x}}-\boldsymbol{\psi}^{T} \frac{\partial \mathbf{R}}{\partial \mathbf{x}} .
$$

We use a Krylov method to solve the linear coupled aerostructural adjoint equations (10) in an analogous manner to the Krylov method applied to the linearized Newton system. In the Krylov approach, the matrix-vector products are computed using the exact Jacobian-transpose of the coupled aerostructural system. One iteration of a transpose block Jacobi iteration is used as the preconditioner. 


\section{The QCRM wing}

For this study we created a wing based on the common research model (CRM) configuration used in the DPW 4 and 5 workshops [32], which we call the Quasi-CRM (QCRM) wing. The QCRM is a wing and wing-tail geometry that has a planform that is roughly the same as the CRM, but the QCRM geometry is not directly derived from the CRM wing.

The QCRM wing has several advantages over the CRM wing for parametric design studies. The QCRM is based on the jig shape of the wing and does not implicitly assume any aeroelastic deflection, as the CRM geometry does. This greatly simplifies aerostructural design studies, since the wing deflections do not have to be removed from the wing geometry. In addition, the QCRM geometry has a simple parametric description, and as a result, the wing planform can easily be manipulated using a small number of geometric design variables. Since the QCRM wing is untwisted, the aerodynamic performance of the wing will be poor. However, we always compare the performance of optimized designs so the initial planform and twist distributions are unimportant.
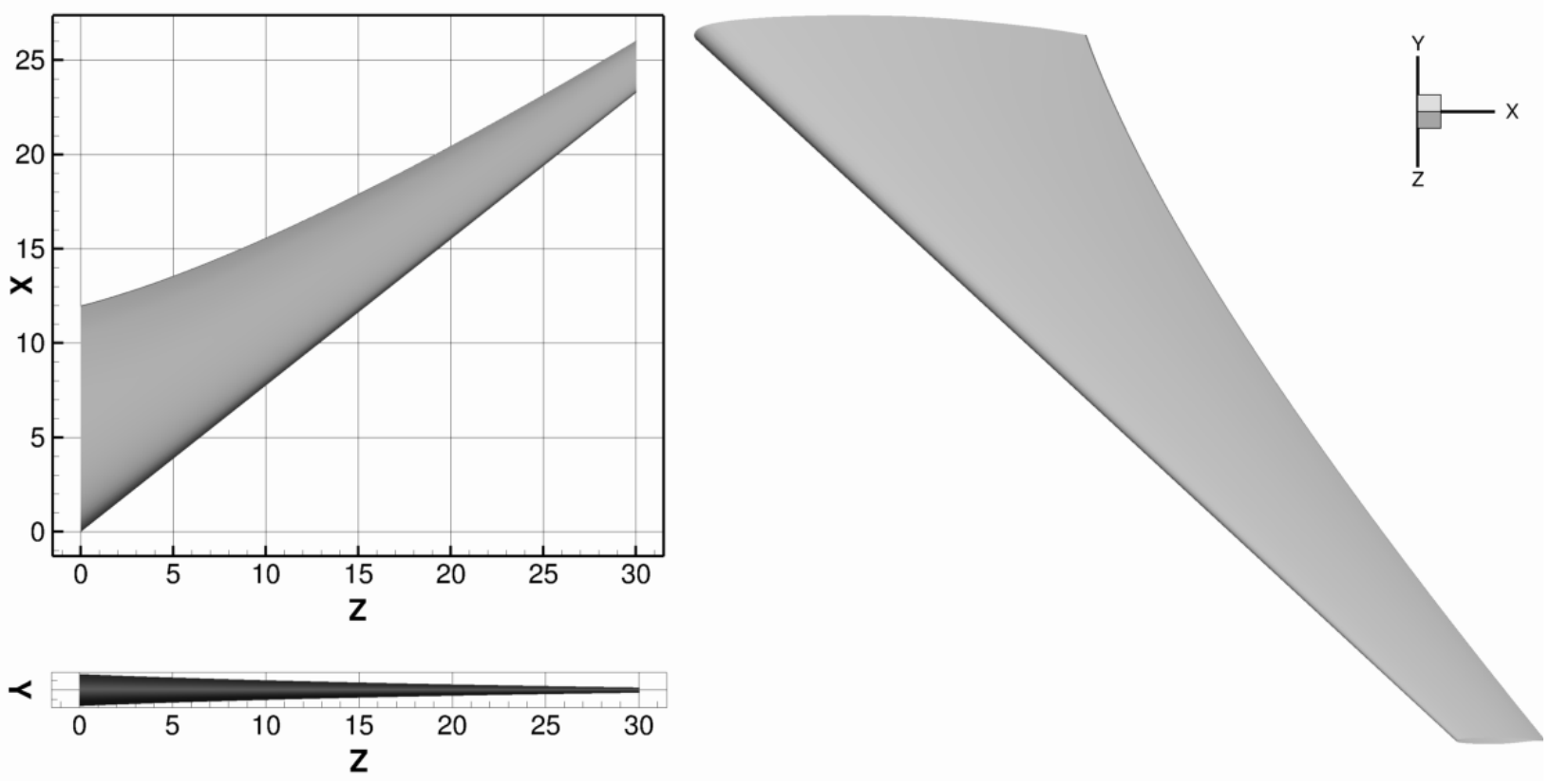

Figure 1: The Quasi-CRM (QCRM) wing geometry is based on the CRM planform and was created to facilitate aerostructural design optimization studies.

The QCRM wing is shown in Figure 1. The QCRM wing is untwisted, has a root chord of $12 \mathrm{~m}$, a tip chord or $2.75 \mathrm{~m}$, a semi-span of $30 \mathrm{~m}$ and a quarter-chord sweep of $35^{\circ}$. The leading edge of the wing is straight, while the trailing edge of the wing is quadratically interpolated between the root trailing edge and the tip. The trailing edge is determined using a quadratic spline where a control point is added at a span-wise location of $10.5 \mathrm{~m}$, with a chord of $6.5 \mathrm{~m}$. The curved trailing edge corresponds roughly with a wing crank, but is entirely smooth. The wing is constructed using a set of lofted super-critical NASA SC(2)-0414 and SC(2)-0610 airfoil sections. While the initial aerodynamic performance of this wing is poor, we do not use its initial performance as a baseline. Instead, we only compare optimized designs.

The internal wing structure geometry is shown in Figure 2. The wing box structure consists of two structural spars and 44 ribs as well as top and bottom wing skins. In addition, non-structural elements are added to the model to improve the load and displacement transfer between the aerodynamic and structural models. These non-structural elements consist of the leading edge skin and a false trailing edge spar and skin that extend behind the true trailing edge spar. A more detailed wing model could include the control surfaces and leading and trailing edge support structures. However, fully modeling these additional features is not considered here due to the additional complexity. Furthermore, it would be necessary to add additional load cases to the design problem to properly size these portions of the structure, complicating the design problem formulation. We have also experimented with the complete omission of the leading and trailing edge surfaces, but this leads to difficulties, as the structural displacements must be extrapolated to the aerodynamic surface. When the structural and aerodynamic surfaces are far apart, the extrapolation of the deformed aerodynamic surface becomes inaccurate, and maintaining a smooth deformed surface can be difficult. As 


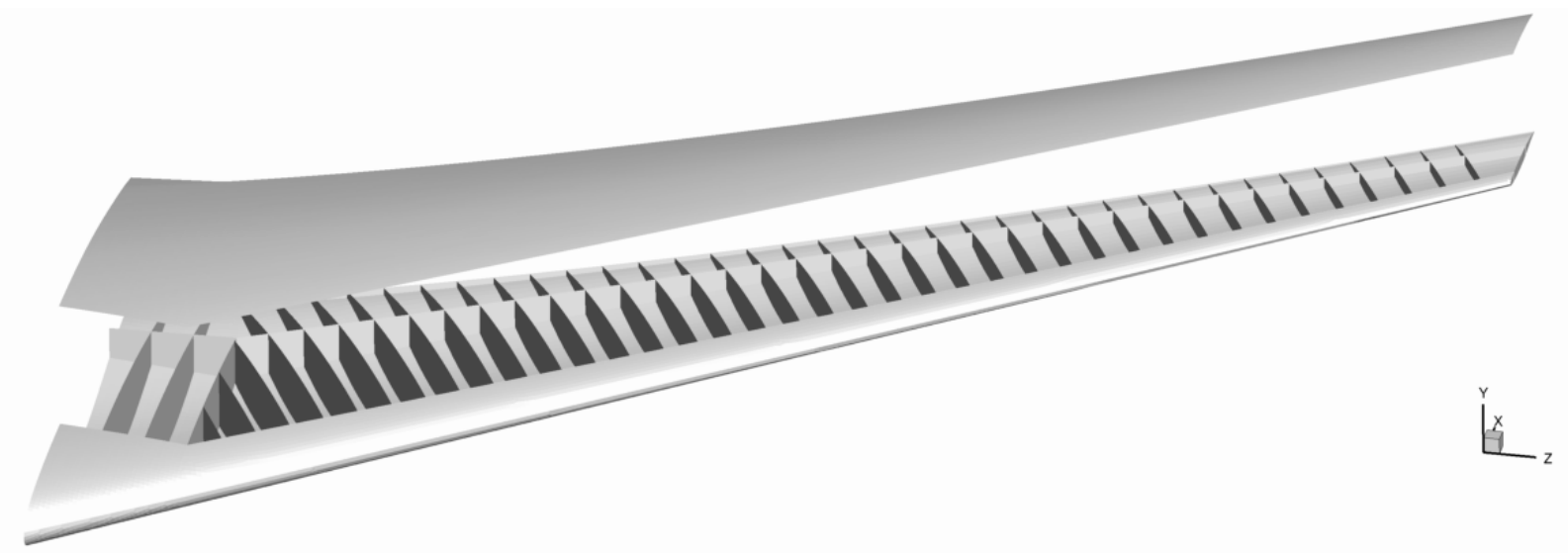

Figure 2: The QRM structural wing box model includes spars, ribs, and skins with smeared stiffeners.

a result, we include these non-structural elements as a compromise. One of the primary purposes of the structural model is to provide an accurate weight estimate based on the primary structure within wing. Therefore, we omit the non-structural components from the weight estimate and set them with a minimum thickness determined by the part type, which is described in more detail below. Furthermore, we reduce the stiffness of these components and maintain a consistent Poisson's ratio to avoid stress concentration effects.

Within the wing box, the first 3 ribs in the structure are parallel to the symmetry plane. This section of the wing box lies within the fuselage and is designed to model the center wing-box. The leading and trailing edge spars lie at $15 \%$ and $65 \%$ of the local chord, respectively, while the non-structural trailing edge extends to $90 \%$ of the chord. The structural finite-element model used for both low and high-fidelity analysis consists of 31,130 third order MITC9 shell finite-elements with 131630 nodes and roughly 789780 degrees of freedom.

\section{Structural design parametrizations}

The parametrization of the structural design defines the design variables that the optimization algorithm can control. In this section, we present the structural parametrizations for the metallic, composite and CNT-based composite wings. We model the structure using a smeared stiffness approach. In the smeared stiffener approach the effect of the stiffeners on the stiffness of the panels is included in the material properties of the panel directly rather than modeling each discrete stiffener individually. This approach works well when the ratio of the panel side-length to stiffener pitch is high, such that there is a high density of stiffeners [30]. Furthermore, the smeared stiffener approach reduces the mesh spacing requirements significantly compared to a discrete stiffener techniques, greatly reducing computational costs associated with the structural analysis.

In the structural parametrization for the metallic, composite and CNT-based composite wings, we split the wing structure into approximately flat panels that are analyzed and designed based on the stress state in the global finiteelement model under a series of loading conditions. These panels consist of the structural components formed between the ribs and spars of the wing. In order to obtain an accurate estimate of the overall wing-box weight it is necessary to have a design tool that can correctly size panels over a wide range of loading conditions. The panels range from relatively lightly loaded at the tip to heavily loaded at the wing root. Over this range, it is most important to capture the behavior of the heavily loaded parts of the structure, since these structural components will have the greatest impact on the structural wing weight.

For the present work, we developed a design tool that predicts the stiffness of the panel based on a smearedstiffener approach. This tool can also be used to enforce strength, buckling, and manufacturing constraints, as well as adjacency constraints that impose limits on the variation of the design variables between adjacent panels. The sizing of the panel is based on the simplified stiffness calculations and buckling criteria proposed by Stroud and Agranoff [30].

To avoid some of the more challenging aspects of composite design problems, we do not design the exact stacking sequence of the structure [13, 12]. Instead, we obtain an approximate design based on the fractions of plies at given angles. Here, we restrict the possible ply angles to $0^{\circ}, \pm 45^{\circ}$ and $90^{\circ}$, where we enforce a balanced laminate by requiring that the ply fractions of the positive and negative $45^{\circ}$ plies are equal. The in-plane strength and stiffness can be determined exactly using the ply fraction variables alone [8]. However, the bending stiffness, bending strength, 
and buckling characteristics of the laminate cannot be determined exactly without knowledge of the laminate stacking sequence. Instead we use conservative estimates of the exact quantities, where possible. Most importantly, these formula approach the exact formula for very thick panels.

\section{Smeared panel stiffness calculations}

The ply fractions of the skin-panel are denoted $f_{i}^{(p)}$, while the ply fractions in the stiffener are denoted $f_{i}^{(s)}$. The stiffness of the panel and stiffener are based on a stiffness weighted by the ply fractions:

$$
\mathbf{Q}^{(p)}=\sum_{i=1}^{4} f_{i}^{(p)} \overline{\mathbf{Q}}\left(\theta_{i}\right), \quad \mathbf{Q}^{(s)}=\sum_{i=1}^{4} f_{i}^{(s)} \overline{\mathbf{Q}}\left(\theta_{i}\right),
$$

where $\theta_{i}=\left\{-45^{\circ}, 0^{\circ}, 45^{\circ}, 90^{\circ}\right\}, \overline{\mathbf{Q}}(\theta)$ is the stiffness in the global axis, and $\mathbf{Q}^{(p)}$ and $\mathbf{Q}^{(s)}$ are the weighted stiffness of the skin and stiffener, respectively. Note, that ply fractions are not independent, and must satisfy the following linear constraints:

$$
\sum_{i=1}^{4} f_{i}^{(p)}=1, \quad \sum_{i=1}^{4} f_{i}^{(s)}=1 .
$$

The stiffness of the overall panel is determined by accounting for the effect of the discrete stiffeners by adding additional bending and shear stiffness to the skin stiffness. The panel stiffness can be determined based on the panel thickness $t_{p}$, the stiffener height $h_{s}$, the stiffener width $t_{w}$, the stiffener pitch $s_{p}$, and the stiffness of the skin and stiffener $\mathbf{Q}^{(p)}$ and $\mathbf{Q}^{(s)}$. The smeared panel stiffness matrices are written as follows:

$$
\begin{aligned}
& \mathbf{A}=t_{p} \mathbf{Q}^{(p)}+\mathbf{A}^{(s)}, \quad \mathbf{B}=\mathbf{B}^{(s)}, \\
& \mathbf{D}=\frac{t_{p}^{3}}{12} \mathbf{Q}^{(p)}+\mathbf{D}^{(s)}, \quad \mathbf{A}_{s}=\mathbf{A}_{s}^{(p)}+\mathbf{A}_{s}^{(s)} .
\end{aligned}
$$

The non-zero components of the matrices $\mathbf{A}^{(s)}, \mathbf{B}^{(s)}, \mathbf{D}^{(s)}$, and $\mathbf{A}_{s}^{(s)}$ are:

$$
\begin{aligned}
\mathbf{A}_{11}^{(s)} & =\frac{E_{s} A_{s}}{s_{p}}, & \mathbf{B}_{11}^{(s)} & =-\frac{h_{s}}{2 s_{p}} E_{s} A_{s}, \\
\mathbf{D}_{11}^{(s)} & =\frac{E_{s}\left(h_{s}^{2} A_{s}+4 I_{s}\right)}{4 s_{p}}, & \mathbf{A}_{s}^{(s)}{ }_{11} & =\frac{5 G_{s} A_{s}}{6 s_{p}},
\end{aligned}
$$

where, $E_{s}=Q_{11}^{s}-\frac{Q_{21}^{s} Q_{12}^{s}}{Q_{66}^{s}}$ is the extension modulus of the stiffener, $A_{s}$ is the area of the stiffener, and $I_{s}$ is the second moment of area of the stiffener.

\section{Panel-level failure analysis}

Each panel in the structure must be constrained such that the response under each loading condition lies within an allowable operational envelope. In this study, we impose this envelope by considering both failure constraints and buckling constraints. For the metallic cases, the failure envelope is modeled using a von Mises stress failure criterion, while for the composite and CNT-based composite cases, the failure envelope is calculated based on a maximum strain failure criterion. This maximum strain failure criterion can be written as follows:

$$
\max \left\{\frac{\epsilon_{1}}{\epsilon_{1_{t}}}, \frac{\epsilon_{1}}{\epsilon_{1_{c}}}, \frac{\epsilon_{2}}{\epsilon_{2_{t}}}, \frac{\epsilon_{2}}{\epsilon_{2_{c}}} \frac{\gamma_{12}}{\gamma_{12_{s}}},-\frac{\gamma_{12}}{\gamma_{12_{s}}}\right\}
$$

where $\epsilon_{1}, \epsilon_{2}$ and $\gamma_{12}$ are the normal, transverse and engineering shear strains in the local ply axis. The constants $\epsilon_{1_{t}}$, $\epsilon_{1_{c}}$ are the maximum allowable tensile and compressive strains along the fiber direction, while $\epsilon_{2_{t}}$ and $\epsilon_{2_{c}}$ are the maximum tensile and compressive strains in the transverse directions, and $\gamma_{12_{s}}$ is the maximum in-plane shear strain allowable. This failure criteria is applied at all ply angles for the outer-most fibers in the skin, and the lowest fiber in the stiffener. This results in 12 separate failure criteria for each point in the panel where the failure constraint is applied. 


\section{Panel-level buckling analysis}

The buckling constraints are imposed by constraining several independent buckling modes including buckling of the skin between stiffeners, buckling of the stiffeners, and overall panel buckling including stiffeners and skins. The overall critical buckling loads are determined based on the approach of Stroud and Agranoff [30]. The skin and stiffener buckling loads are determined by assuming that the panel ends are simply supported along the lines of attachment with adjacent structural components. The critical loads are determined under the assumption that the panels are nearly flat, and therefore the analysis ignores any curvature effects.

We assume that the interaction between the longitudinal and shear buckling modes collapses into the following buckling envelope:

$$
B\left(N_{1}, N_{12}\right)=\frac{N_{12}^{2}}{N_{12, c r}^{2}}+\frac{N_{1}}{N_{1, c r}} \leq 1,
$$

where $N_{1}$ and $N_{12}$ are the longitudinal and shear loads respectively and $N_{1, c r}$ and $N_{12, c r}$ are the critical longitudinal and shear buckling loads. Note that $N_{1}$ and $N_{12}$ are computed in a locally aligned panel axis. Equation (14) is applied separately to the overall panel buckling, stiffener buckling and inter-stiffener skin buckling. The critical loads, $N_{1, c r}$ and $N_{12, c r}$ are determined based on the formula presented in Table 1.

\begin{tabular}{lll}
\hline & Overall buckling & Skin buckling \\
\hline$N_{1, c r}$ & $\frac{\pi^{2} E I_{s}}{s_{p} L_{x}^{2}}$ & $\frac{2 \pi^{2}}{s_{p}^{2}}\left(\sqrt{D_{11} D_{22}}+D_{12}+2 D_{66}\right)$ \\
$E I_{s}=z_{n}^{2}\left(t_{p} s_{p} E_{p}+t_{s} h_{s} f_{b} E_{s}\right)+E_{s}\left(t_{s} \frac{h_{s}^{3}}{12}+t_{s} h_{s}\left(z_{n}-\frac{h_{s}}{2}\right)^{2}\right)$ \\
$z_{n}=\frac{C_{n}}{A_{n}}$ & $C_{n}=E_{s} \frac{h_{s}^{2}}{2} t_{s}$ & $A_{n}=E_{p} s_{p} t_{p}+E_{s} h_{s} t_{s}\left(1+f_{b}\right)$ \\
\hline$N_{12, c r} \quad \xi=\frac{\sqrt{D_{1} D_{2}}}{D_{3}}$ & $\xi=\frac{\sqrt{D_{11} D_{22}}}{D_{12}+2 D_{66}}$ \\
If $\xi>1$ & $\frac{4}{L_{x}^{2}}\left(D_{1}^{3} D_{2}\right)^{0.25}\left(8.125+\frac{5.045}{\xi}\right)$ & $\frac{4}{s_{p}^{2}}\left(D_{11} D_{22}^{3}\right)^{0.25}\left(8.125+\frac{5.045}{\xi}\right)$ \\
If $\xi \leq 1$ & $\frac{4}{L_{x}^{2}} \sqrt{D_{1} D_{3}}\left(11.7+0.532 \xi+0.938 \xi^{2}\right)$ & $\frac{4}{s_{p}^{2}} \sqrt{D_{22}\left(D_{12}+2 D_{66}\right)}\left(11.7+0.532 \xi+0.938 \xi^{2}\right)$ \\
\hline
\end{tabular}

Table 1: A summary of the critical load computations for the overall and skin buckling constraints. Note that $A_{n}$ and $C_{n}$ are the modulus-weighted zeroth and first moments of area of the panel and stiffener, and $z_{n}$ is the modulusweighted centroid. The bending stiffness $E I_{s}$ can then be used to determine the critical buckling load.

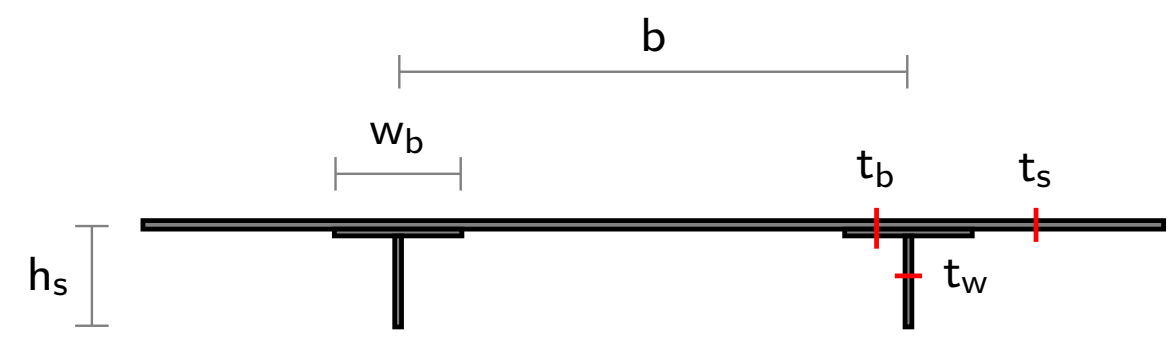

Figure 3: The panel geometry and thickness design variables used in the structural design parametrization. 


\section{Aerostructural design optimization formulation}

The aerostructural design optimization problem is solved using the multidisciplinary feasible MDO architecture [24], whereby each design iteration is feasible with respect to the coupled governing equations, and all design variables are optimized simultaneously in a monolithic fashion. In the aerostructural design problem, we implemented a representative aircraft design problem formulation that captures the most essential aspects of the aircraft design problem in order to quantify the tradeoff between aerodynamic performance and structural weight reduction. This is essential in order to make a fair comparison between potential benefits of candidate structural technologies.

In this design formulation, we minimize a weighted combination of fuel burn and take-off gross weight (TOGW) for a single long-range high-payload mission. We compute the fuel consumption of the mission using the Breguet range equation:

$$
\mathrm{FB}=\mathrm{LGW}\left(\exp \left(\frac{R \mathrm{TSFC}}{V(L / D)}\right)-1\right)
$$

where FB is the fuel burn for the entire mission, LGW is the landing gross weight of the aircraft (which is the sum of the reserve fuel and the zero fuel weight of the aircraft), $R$ is the mission range, TSFC is the thrust-specific fuel consumption, $V$ is the cruise speed, and $L / D$ is the lift-to-drag ratio.

Due to aerostructural effects, the deformed flying shape, and aerodynamic performance of the wing changes with aircraft weight, and thus $L / D$ varies during the mission, even at a fixed lift coefficient. A more accurate analysis would integrate the true aircraft $L / D$ over the mission profile using the Breguet range integral. In this work, however, we neglect these aerostructural effects and instead evaluate the $L / D$ of the aircraft at the half fuel weight condition. Note that this is not the same point as the mid-range point.

To compare the impact of structural weight reduction and fuel burn reduction, we utilize a composite objective that includes both takeoff gross weight and fuel consumption. This provides a more realistic range of designs than the more extreme discipline-level objectives of structural weight and aerodynamic drag. The composite objective can be written as follows:

$$
f(\mathbf{x})=\beta \mathrm{FB}+(1-\beta) \mathrm{TOGW},
$$

for $\beta \in[0,1]$. Note that since the TOGW is the sum of the landing gross weight (LGW) and the fuel burn, this objective is equivalently $f(\mathbf{x})=(2-\beta) \mathrm{FB}+(1-\beta) \mathrm{LGW}$. Alternatively, using Breguet range equation, the objective can be written as follows:

$$
f(\mathbf{x})=\operatorname{LGW}\left(\exp \left(\frac{R \text { TSFC }}{V(L / D)}\right)-\beta\right) .
$$

\section{A. Aerostructural optimization problem description}

\section{Objective function}

The aircraft sizing parameters are based roughly on a next-generation 777-sized aircraft. In an effort to simplify the design problem, only a wing is modeled and constant drag factors are used to estimate the drag of the remaining aircraft components.

We estimate the landing weight, LGW, at the end of the cruise segment using the following expression:

$$
\mathrm{TOGW}=\mathrm{OEW}_{\text {fixed }}+K_{1} \times M_{\text {struct }}+K_{2} \times A_{\text {planform }}+M_{\text {reserve }}+M_{\text {payload }}
$$

The $\mathrm{OEW}_{\text {fixed }}$ is derived from the preliminary aircraft design structural weight breakdown given by Kroo [16]. For the metallic design this results in a fixed OEW of $98700 \mathrm{~kg}$. For the composite and CNT designs, we assumed a weight savings of $30 \%$ for the fuselage, horizontal stabilizer and vertical stabilizer and this results in a fixed OEW that is $10500 \mathrm{~kg}$ lower than the metallic case, or $\mathrm{OEW}_{\text {fixed }}=88200 \mathrm{~kg}$.

The structural mass, $M_{\text {struct }}$, is evaluated directly from the finite-element model. A mark-up factor $K_{1}=1.5$ is applied to account for additional fastener weight and structural component that are absent from the finite-element model. There is an additional contribution, $K_{2} \times A_{\text {planform }}$ which is designed to account for the weight contributions from the leading and trailing edge wing structures. For the optimization presented, we use $K_{2}=15 \mathrm{~kg} / \mathrm{m}^{2}$.

The reserve fuel is calculated based on a 45 minute loiter at sub-optimal $L / D$ and TSFC conditions, and a diversion to an alternate airport $500 \mathrm{~nm}$ away. For simplicity, the resulting reserve fuel quantity, $14100 \mathrm{~kg}$, remains fixed throughout the optimization.

The cruise mission uses the maximum payload, maximum range mission for the 777-200ER aircraft. The maximum payload is set at $55000 \mathrm{~kg}$ and the mission range is $6000 \mathrm{~nm}$. The $L / D$ performance of the aircraft is evaluated at the point in the mission where half of the mission fuel has been consumed. To account for the drag on the fuselage, 
horizontal stabilizer, vertical stabilizer and nacelles, a constraint drag factor of 0.0100 is added the total drag computed on the QCRM wing geometry:

$$
\frac{L}{D}=\frac{C_{L}}{C_{D}+0.0100}
$$

A summary of the relevant parameters is given in Table 2 .

\begin{tabular}{lr}
\hline Parameter & Value \\
\hline Fixed O.E.W. (metallic) & $98700 \mathrm{~kg}$ \\
Fixed O.E.W. (composite) & $88200 \mathrm{~kg}$ \\
$K_{1}$ & 1.5 \\
$K_{2}$ & $15 \mathrm{~kg} / \mathrm{m}^{2}$ \\
Reserve Fuel & $14100 \mathrm{~kg}$ \\
Payload & $55000 \mathrm{~kg}$ \\
Range & $6000 \mathrm{~nm}$ \\
Analysis altitude & $36000 \mathrm{ft}$ \\
Mach & 0.85 \\
Span & $60 \mathrm{~m}$ \\
$C_{D_{0}}$ & 0.0100 \\
Root chord & $12 \mathrm{~m}$ \\
Tip chord & $2.75 \mathrm{~m}$ \\
Leading Edge Sweep & $37.2^{\circ}$ \\
$S_{r e f}$ & $397.46 \mathrm{~m}^{2}$ \\
Maneuver Altitude & $12000 \mathrm{ft}$ \\
Maneuver Mach & 0.75 \\
\hline
\end{tabular}

Table 2: Parameters used for the aircraft design problem.

Table 3 shows the properties of the materials used in this study including the metallic, composite and CNT-based composite. Note that the hypothetical CNT-based composite material is based on a tensile modulus of $1.2 \mathrm{TPa}$ and a tensile strength of $6 \mathrm{GPa}$, while the remaining properties are scaled to match the corresponding composite material data. A composite material with such properties is currently in the realm of science fiction, but we use it here to quantify the impact on wing design of improving material properties by an order of magnitude.

\section{Design Constraints}

The optimization problems presented require a variety of constraints to ensure a physically meaningful designs.

For the aerostructural problem, we use a series of failure and buckling constraints that are imposed at two critical points within the design envelope. These are the $2.5 \mathrm{~g}$ and $-1 \mathrm{~g}$ points shown in the V-n diagram of Figure 4 . For both the failure and buckling constraints we employ a Kreisselmeier-Steinhauser (KS) aggregation technique [28], where we group point-wise constraints from within each element into a small number of global constraints. The KS function can be written as follows:

$$
\mathrm{KS}=c_{\max }+\frac{1}{\rho_{K S}} \ln \left[\sum_{i=1}^{N} \exp \left(\rho_{K S}\left(c_{i}-c_{\max }\right)\right)\right],
$$

where $c_{i}$ are all the point-wise failure or buckling constraints in the aggregation domain, $c_{\max }=\max \left\{c_{1}, c_{2}, \ldots, c_{N}\right\}$ is the maximum value of the failure or buckling constraint, and $\rho_{K S}$ is a penalty parameter that we set to 80.0 in all calculations presented here. Since the KS function is conservative, if the failure envelope of the KS function is not violated, then none of the individual element-wise failure functions is violated. Instead of aggregating all the failure and buckling functions into a single constraint, we group the failure and buckling constraints separately over common structural components. We separate the top and bottom wing skins, respectively and group all spars and ribs into a single KS constraint. Since we do not utilize buckling constraints for the spars and ribs, there are only two KS buckling constraints, while there are three KS failure constraints for each structural load condition. 


\begin{tabular}{|c|c|c|c|c|c|}
\hline Parameter & Value & Units & Parameter & Value & Units \\
\hline \multicolumn{6}{|c|}{ Aluminum material data } \\
\hline$E$ & 70.0 & $\mathrm{GPa}$ & $\nu$ & 0.3 & \\
\hline$\sigma_{Y S}$ & 420 & $\mathrm{MPa}$ & $\rho$ & 2780 & $\mathrm{~kg} / \mathrm{m}^{3}$ \\
\hline \multicolumn{6}{|c|}{ Composite material data } \\
\hline$E_{1}$ & 128 & $\mathrm{GPa}$ & $E_{2}$ & 11 & $\mathrm{GPa}$ \\
\hline$G_{12}$ & 4.5 & $\mathrm{GPa}$ & $G_{13}$ & 4.5 & $\mathrm{GPa}$ \\
\hline$G_{23}$ & 3.2 & $\mathrm{GPa}$ & $\nu_{12}$ & 0.25 & \\
\hline$X_{t}$ & 1170 & $\mathrm{MPa}$ & $X_{c}$ & 1120 & $\mathrm{MPa}$ \\
\hline$Y_{t}$ & 40 & $\mathrm{MPa}$ & $Y_{c}$ & 170 & $\mathrm{MPa}$ \\
\hline$S$ & 48 & $\mathrm{MPa}$ & $\rho$ & 1522 & $\mathrm{~kg} / \mathrm{m}^{3}$ \\
\hline \multicolumn{6}{|c|}{ Carbon nanotube-based composite material data } \\
\hline$E_{1}$ & 1200 & $\mathrm{GPa}$ & $E_{2}$ & 120 & $\mathrm{GPa}$ \\
\hline$G_{12}$ & 45 & $\mathrm{GPa}$ & $G_{13}$ & 45 & $\mathrm{GPa}$ \\
\hline$G_{23}$ & 32 & $\mathrm{GPa}$ & $\nu_{12}$ & 0.25 & \\
\hline$X_{t}$ & 6000 & $\mathrm{MPa}$ & $X_{c}$ & 5000 & $\mathrm{MPa}$ \\
\hline$Y_{t}$ & 400 & $\mathrm{MPa}$ & $Y_{c}$ & 1600 & $\mathrm{MPa}$ \\
\hline$S$ & 500 & $\mathrm{MPa}$ & $\rho$ & 1522 & $\mathrm{~kg} / \mathrm{m}^{3}$ \\
\hline
\end{tabular}

Table 3: Mechanical properties of the metallic, conventional composite and hypothetical CNT-based composite used in this study.

\section{Design Variables}

The design variables consist of geometric variables, structural variables, aerodynamic design variables and consistency variables. The aerodynamic design variables consist of the angles of attack at each of the cruise and maneuver conditions. However, to avoid inconsistencies resulting from both root twist and angle of attack, the cruise alpha remains fixed.

For the TriPan analysis, the geometric variables consists of 8 twist variables distributed along the wing span, one span-scaling variable, and one chord scaling variable and 8 vertical scaling variables, which modify the thickness to chord ratio. Together the span and chord scaling variables admit a series of planforms that are stretched in the chord-wise and span-wise directions, but share similar geometric features.

For the CFD-based design optimization, the same twist, span and chord variables are used, and 192 airfoil shape variables are added. The shape variables control the airfoil shapes and permit arbitrary thickness distributions in both the chord and spanwise direction, and thus the vertical scaling variables used with TriPan are not required. A view of the free form deformation volume used for the CFD-based design optimization is given in Figure 5.

The structural design variables are the same for both the low and high fidelity analysis. Two variables determine the stiffener pitch of the entire upper and lower skins, respectively. Each panel formed by the leading edge, trailing edge, and ribs is assigned the variables shown in Figure 3. Each panel has two thickness variables for the skin and stiffener, respectively, as well as a panel height. The stiffener base width is fixed based on the stiffener height. For the metallic cases, no further information is necessary. For the composite and CNT designs there are three additional variables that define the ply-fraction for the skin. In this work, we fix the ply-fractions within the stiffeners to a $0^{\circ}$-ply dominant laminate.

There are several consistency variables that are required in the design problem. First, we add a fuel mass consistency variable for each of the 4 missions. These variables are used in the application of the inertial relief from the fuel loads. They ensure that a fraction of the total fuel load is applied to the wing. In addition, we add an equivalent fuel traction on either the top or bottom skins for each maneuver load. These loads correspond to the surface force per-unit-area exerted by the fuel on the wing. We add a consistency variable for each rib-bay for the entire wing. Finally, for the buckling constraints, it is necessary to have an equivalent panel length, since this information cannot be calculated for each element, we add a length variable for each panel in the wing. We then add a geometric constraint that the physical panel length must match the panel length variable for each component of the wing. 


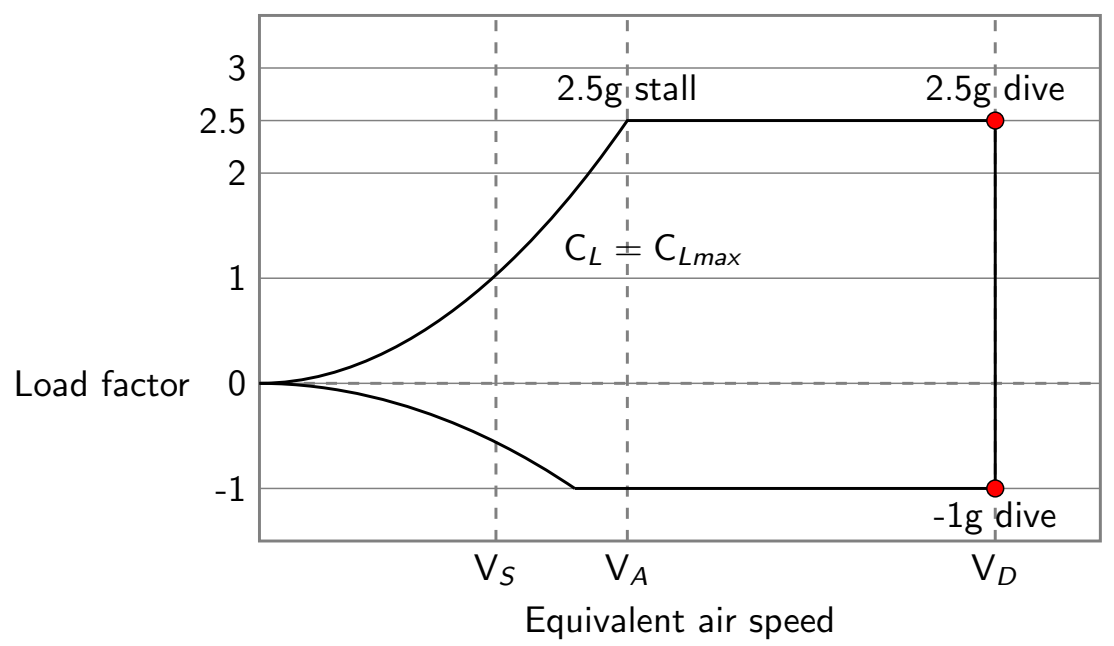

Figure 4: V-n diagram showing the critical points where the structural constraints are imposed.

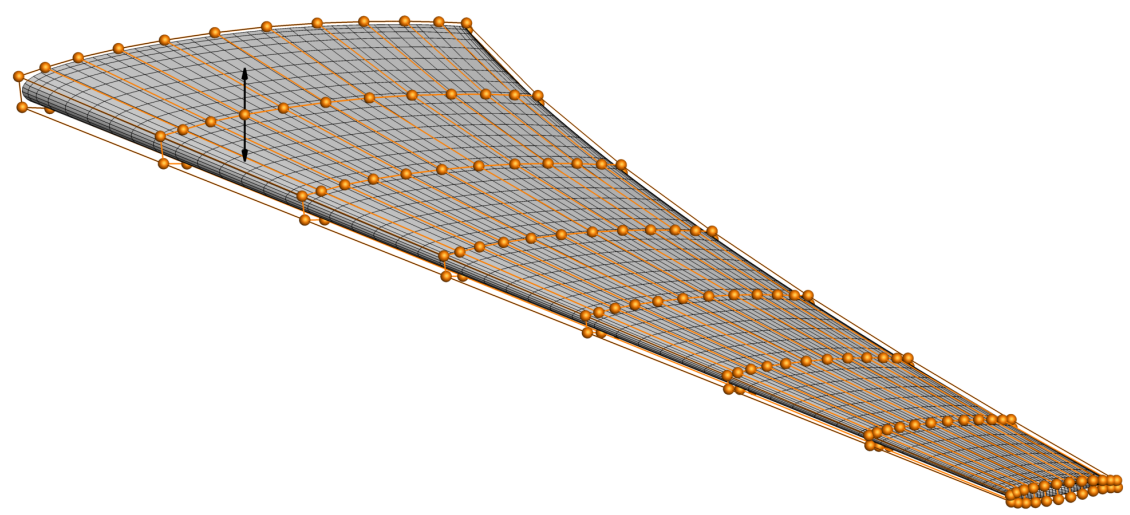

Figure 5: The design variables for the CFD-based design optimization are the vertical positions of 192 points that control the wing shape through a free form deformation (FFD) volume.

\section{Optimization Problem Statement}

The aerostructural optimization problem can now be summarized as follows: The objective is to minimize a linear combination of the TOGW and fuel burn by varying the external shape variables and structural sizing variables described above, as well as the ply fractions when the material is a composite. Simultaneously, the structural design must satisfy a series of failure and buckling constraints at the two off-design conditions shown in Figure 4 such that the three KS failure constraints and two KS buckling constraints at each maneuver load are within the admissible failure envelope. In addition, we impose steady-state lift and trim constraints at each of the 3 operating conditions. 
The aerostructural optimization problem can be written as follows:

\begin{tabular}{|c|c|c|}
\hline minimize & $\beta \mathrm{FB}+(1-\beta) \mathrm{TOGW}$ & \\
\hline w.r.t. & $\mathrm{x}$ & \\
\hline \multirow[t]{5}{*}{ such that } & $\mathrm{KS}_{\text {fail }}^{(i)} \leq 1$ & $i=2, \ldots, 3$ \\
\hline & $\mathrm{KS}_{\text {buckling }}^{(i)} \leq 1$ & $i=2, \ldots, 3$ \\
\hline & $L^{(i)}=n^{(i)} W^{(i)}$ & $i=1, \ldots, 3$ \\
\hline & $\mathbf{c}(\mathbf{x})=0$ & \\
\hline & $\mathbf{l} \leq \mathbf{A x} \leq \mathbf{u}$ & \\
\hline
\end{tabular}

where $\mathrm{KS}_{\text {fail }}^{(i)}$ and $\mathrm{KS}_{\text {buckling }}^{(i)}$ are the $\mathrm{KS}$ failure and $\mathrm{KS}$ buckling constraints, $L^{(i)}=n^{(i)} W^{(i)}$ and is the lift constraint for each analysis condition, and $n_{(i)}$ is the load factor, $\mathbf{c}(\mathbf{x})=0$ represents all the consistency constraints and $\mathbf{l} \leq \mathbf{A x} \leq \mathbf{u}$ represents the adjacency constraints. Note that the consistency constraints are nonlinear but do not depend on the solution of the aerostructural problem directly, therefore their derivatives are inexpensive to evaluate.

\section{Results and Discussion}

In this section, we present the results from a series of optimizations for the metallic, conventional composite and CNT-based composite wings. We present results for both the medium- and RANS-based high-fidelity aerostructural optimization. The conclusions drawn from both sets of optimizations are helpful in assessing the impact of structural technologies on the tradeoff between structural weight reduction and drag minimization. As part of this study, we present a series of optimizations with different values of the parameter $\beta$ using the aerostructural design formulation (21). In addition, we compare the fully integrated designs we obtain with the full aerostructural optimization framework, to a sequential design approach. In the sequential approach, we repeatedly perform design iterations where the structure is sized at fixed aerodynamic loads to obtain a structural weight estimate, followed by an aerodynamic optimization at fixed structural weight to minimize the fuel burn. This design process ignores the impact of aeroelastic deformation, but provides a useful comparison of the fully integrated optimization approach with a sequential design method.

\section{A. Medium-fidelity aerostructural optimization}

First, we present the results from the aerostructural optimization framework that uses the panel-method aerodynamics with empirical profile and wave drag corrections. The results from this study help inform the interpretation of the full RANS-based high-fidelity aerostructural optimization results and provide insight into the most important aspects of the problem.

Figure 6 shows the planforms of the aerostructural optimization results for the metallic, conventional composite and CNT-based composite designs. Both the metallic and conventional composite designs are shown for $\beta=0,0.5$, $0.625,0.75,0.875$, and 1 , while the CNT-based results are shown for $\beta=0,0.5,0.75$, and 1 . For all designs the spans increase monotonically as $\beta$ increases. This trend reflects the increasing importance placed on fuel burn reduction as reflected in the objectives for larger values of $\beta$. While all designs exhibit increasing aspect ratios, the aspect ratios for the conventional composites and CNT-based composites, are higher than conventional aircraft.

Figure 7 shows the Pareto fronts for the designs obtained from the aerostructural optimization with the various values of $\beta$. Recall that $\beta=0$ corresponds the a TOGW minimization, $\beta=1$ corresponds the a fuel burn minimization, and intermediate values represent a compromise between these two objectives. Figure 7 also show the results from a sequential design method for a series of fixed spans with metallic wings. As we can see, there is a significant difference between the performance of the wing obtained from a sequential design method and the aerostructural optimization results. The performance for the sequential designs is predicted using aerodynamic analysis only, without the impact of aeroelastic optimization. Therefore, the actual performance of the designs will be different than what is shown here. We are currently working to refine the sequential design method in order to better reflect current industrial practice. The sequential designs exhibit higher takeoff gross weights than all but the $\beta=1$ aerostructural fuel burn optimization. These sequential optimization results are in agreement with previous low-fidelity aerostructural optimization results, where the sequential approach was shown to be inferior relative to the MDO approach [5, 3]. The present study is the first such comparison for higher-fidelity models, and it is also the first comparison of a Pareto front obtained from sequential optimization. 


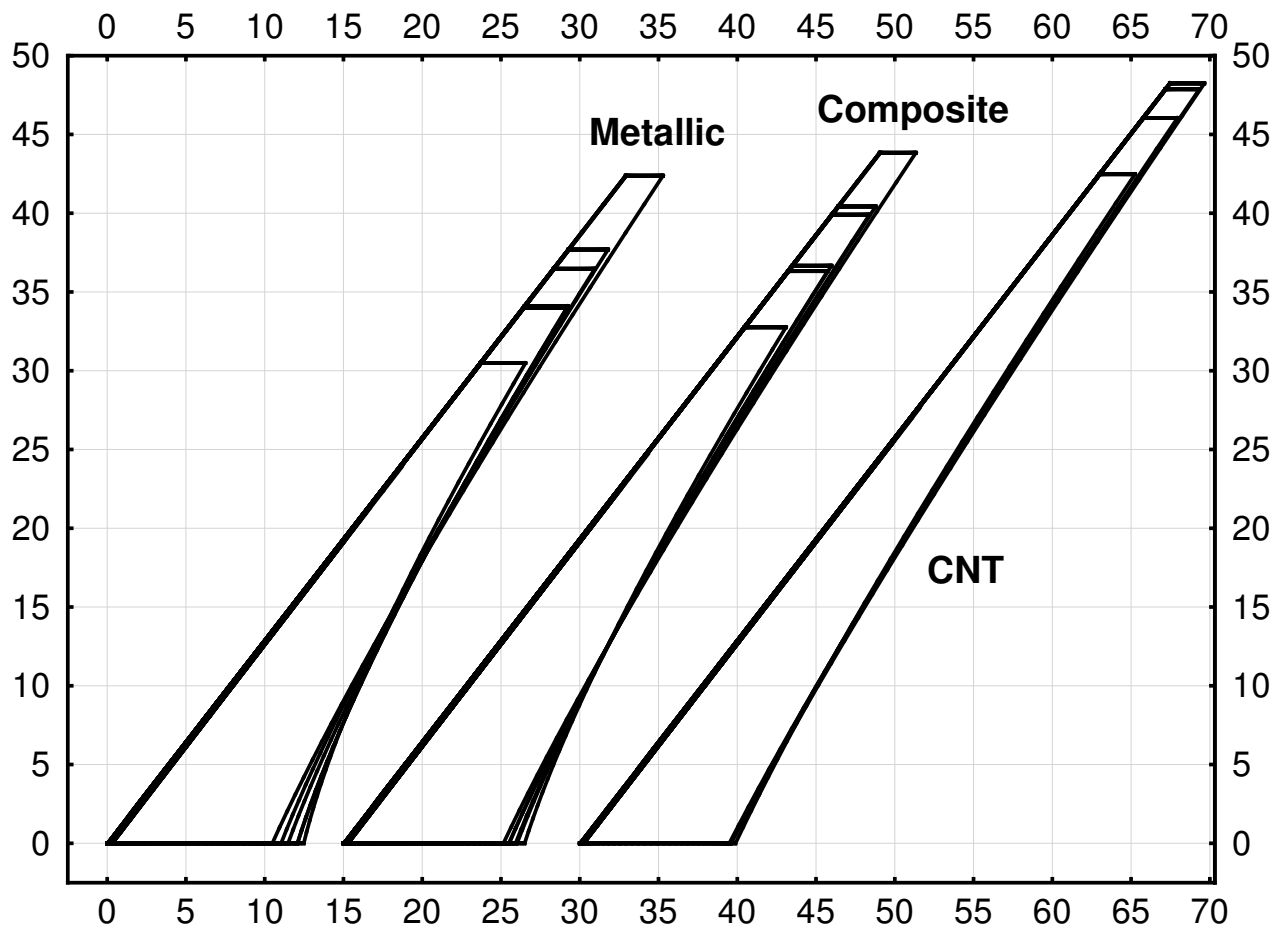

Figure 6: The planforms for the metallic, conventional composite and CNT-based composite wings show that aerostructurally optimal spans increase as the material properties improve.

From the Pareto front trends shown in Figure 7 it is clear that the use of advanced materials leads to simultaneous fuel burn and structural weight improvements. As more advanced materials are used, the difference in performance between fuel burn minimization and TOGW minimized designs becomes smaller and the Pareto fronts become more compact. This is due to the larger weight savings, which reduces both fuel consumption and TOGW directly. The composite TOGW design $(\beta=0)$ is $8.4 \%$ lighter than the metallic baseline, and the CNT-based composite design is $5.6 \%$ lighter relative to the $\beta=0$ composite design. For the fuel burn optimized designs $(\beta=1)$ the composite design exhibits a $7.7 \%$ fuel burn advantage compared to the metallic design, and the CNT-based composite exhibits a further $5.2 \%$ fuel burn reduction compared to the composite $\beta=1$ design. Thus, the improvements in performance of the CNT-based composite wings are relatively modest compared to the drastically improved performance assumed in the material properties. This is in part because the weight savings were limited by minimum thickness constraints, as we will see later.

Figure 8 shows the fuel burn and TOGW as a function of span for the metallic, conventional composite and CNTbased composite designs, as well as the sequential design optimization method. The fuel burn optimized designs exhibit large spans (all greater than $80 \mathrm{~m}$ ) at the cost of increased wing structural weight, which explains the TOGW trend. The combination of the fuel burn and TOGW trend with span demonstrate the key tradeoff that makes aerostructural design optimization so challenging: The objective function is very flat with respect to span, since the changes in structural weight are balanced by the induced drag [17]. A flat objective function makes it difficult to differentiate between designs with different spans, and numerical issues arise when using optimization to find the best design.

Figure 9 shows the cruise $L / D$ as a function of the aspect ratio for the metallic, conventional composite, and CNTbased composite designs. The metallic designs exhibit aspect ratios between 9.4 and 15.5, the conventional composite designs exhibit aspect ratios between 10.9 and 16.5, and the CNT-based composite wings exhibit aspect ratios between 16.6 and 19.5. The RANS-based high-fidelity wing results exhibit aspect ratios of around 11. Nearly all the medium fidelity results exhibit the same trend: increasing aspect ratio and $L / D$ as more emphasis is put on minimizing fuel burn (increasing $\beta$ ). Another key trend is the variation of cruise $L / D$ both with fixed structural technology and between different structural technologies. The cruise $L / D$ increases monotonically for fixed wing construction as more emphasis is put on minimizing fuel burn. This is expected since fuel burn reduction can be achieved by increasing the $L / D$ at cruise. However, between different structural technologies, the cruise $L / D$ decreases, despite the significant 7.7\% and 5.4\% fuel burn reductions between the metallic and composite, and composite and CNT designs shown in 


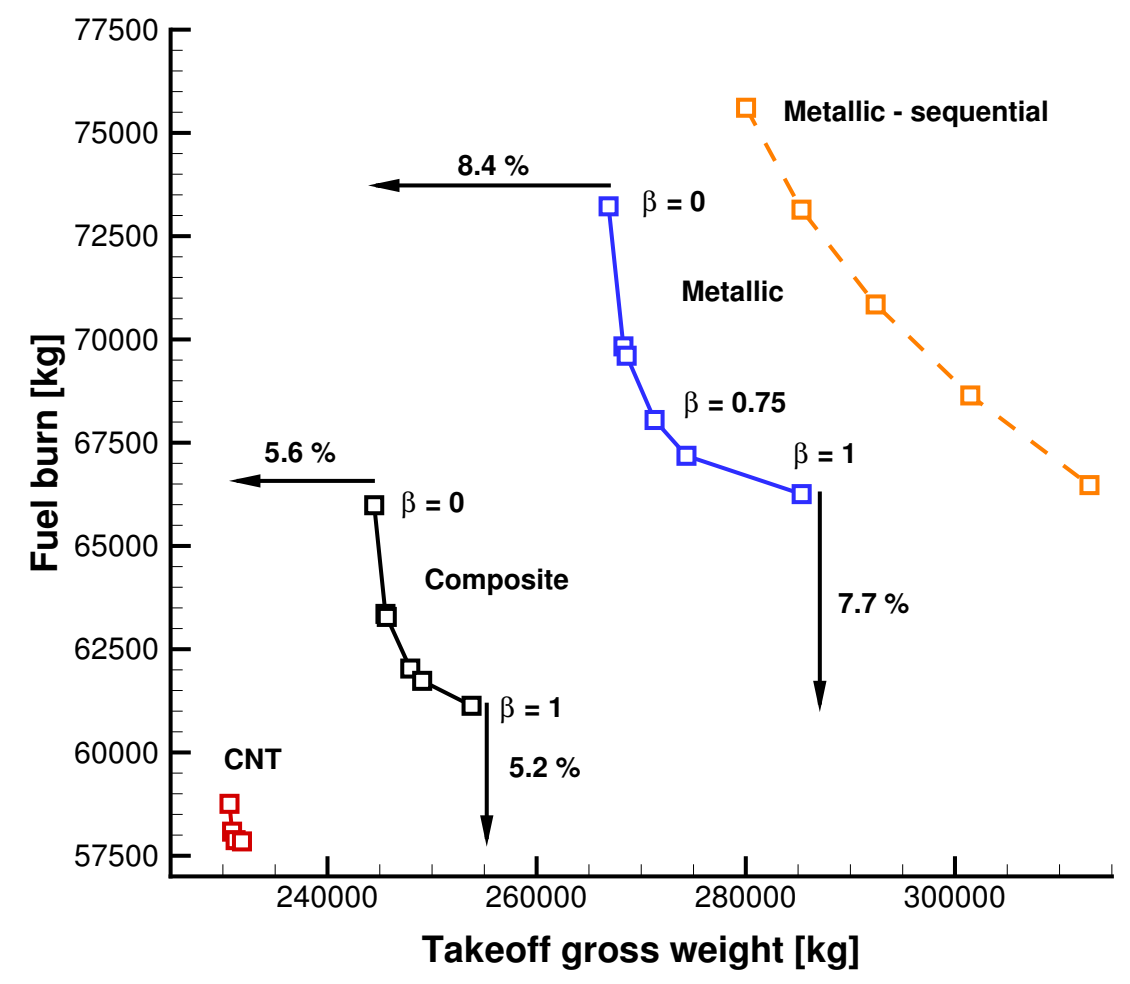

Figure 7: The Pareto fronts of fuel burn and takeoff gross weight shows the advantage of the conventional composite and CNT composite materials, as well as the advantage of aerostructural design optimization over sequential optimization.
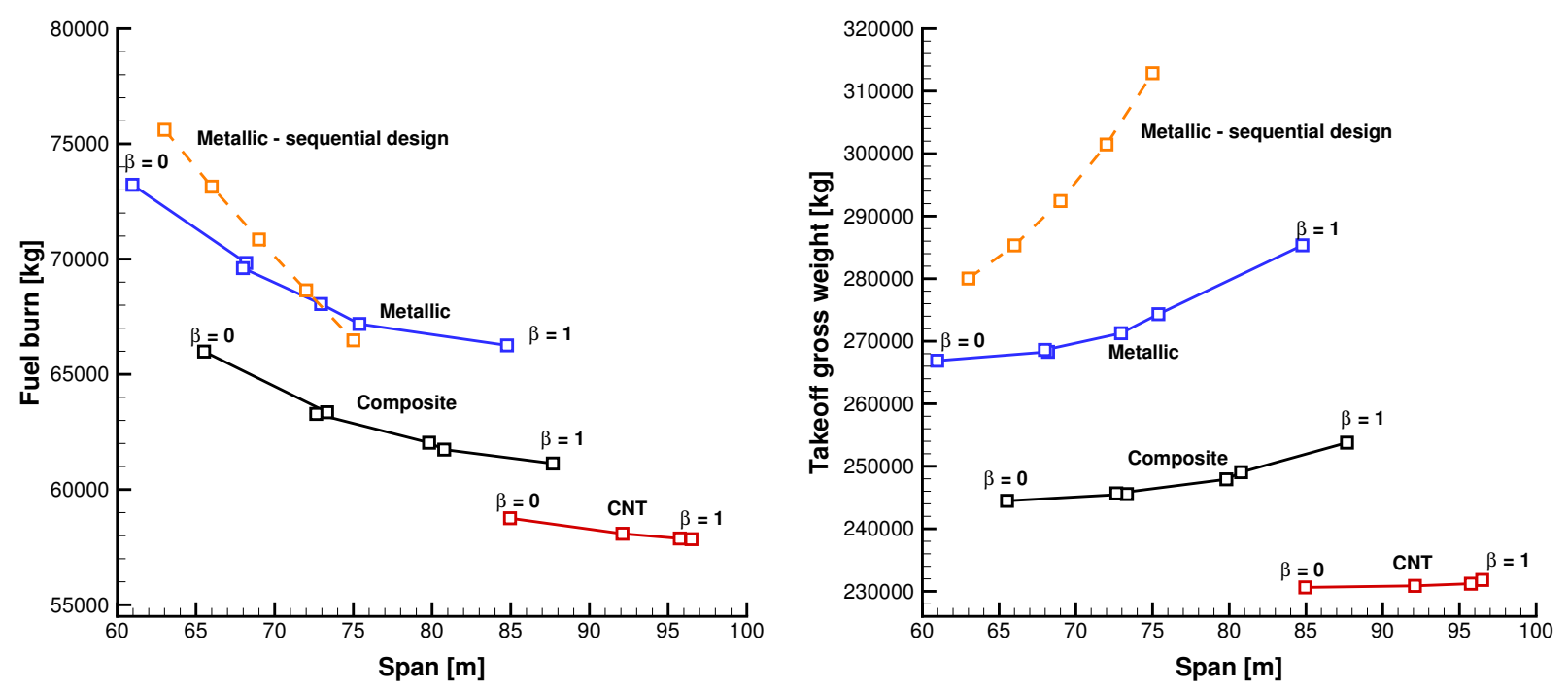

Figure 8: The fuel burn and MTOW as a function of the span for the metallic, conventional composite and CNT-based composite wings.

Figure 7. A preliminary analysis of the results suggests that further fuel burn reductions could be achieved by allowing the designs with larger wing areas to fly at higher altitudes wither more optimal $L / D$ values. This altitude variation 


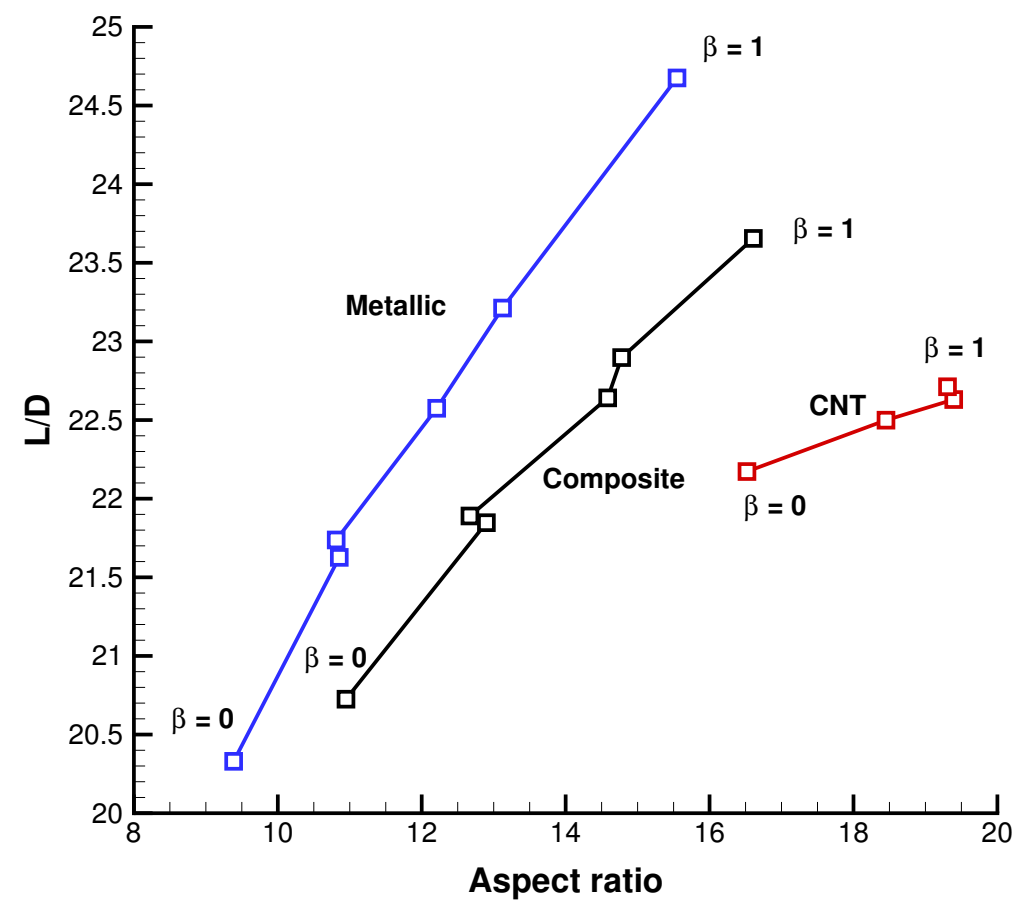

Figure 9: $L / D$ performance of the metallic, conventional composite and CNT-based wings plotted against aspect ratio.

would enable further fuel burn reduction. In future, we will also assess the impact of enabling altitude variation during the design optimization, which could impact the final design.

Figure 10 shows the twist distributions at the cruise and $2.5 \mathrm{~g}$ maneuver conditions for the metallic, conventional composite and CNT-based composite designs. All designs exhibit significant aeroelastic twist and passive maneuver load alleviation. The lift distributions in the cruise condition exhibit more elliptical lift distributions wither higher tip loads, while the wing loading for the $2.5 \mathrm{~g}$ maneuver condition is shifted inboard significantly. Both the metallic and composite designs exhibit roughly the same tip twist (within $1^{\circ}$ ). The CNT-based composite designs exhibit less tip twist than either the composite designs due to the significantly higher stiffness of the CNT-based composite.

Figure 11 shows the skin thickness distributions for the top and bottom skins of the metallic, conventional composite and CNT-based composite designs. The panel thickness for the metallic design shown in Figure 11a exhibits slightly larger thicknesses for the lower skin compared to the upper skin. For the composite designs, the lower skin thickness is significantly higher than the upper skin thickness due to a combination of the buckling and failure criteria at the $-1 \mathrm{~g}$ condition and the failure criterion at the $2.5 \mathrm{~g}$ condition. Note that the designs for each of the metallic, conventional composite and CNT-based composite exhibit consistent design trends between the TOGW $(\beta=0)$ and fuel burn $(\beta=1)$ objectives.

Figure 12 shows the ply fractions in the wing skins for the composite and CNT-based designs. The reference axis associated with the ply angles is aligned along leading edge span of the wing. For both the composite and CNT-based composite wings, the designs with larger wing spans incorporate greater fractions of $0^{\circ}$ plies. The spanwise variation of the ply fractions is also significant. All designs tend to utilize greater fractions of $0^{\circ}$ plies near the root and larger fractions of $\pm 45^{\circ}$ plies towards the wing tip. This design feature reduces the tip twist of the wing under load and offsets the loss of torsional stiffness from the smaller, thinner tip sections.

\section{B. RANS-based aerostructural optimization}

We now investigate two of the metallic design optimization problems using the RANS-based aerostructural optimization. Two optimizations at the extremes of the Pareto front are performed: one for TOGW $(\beta=0)$ and one for fuel burn $(\beta=1)$. The objective is to identify differences in the resulting designs that are primarily caused by the higher fidelity aerodynamic analysis and the additional flexibility introduced through the airfoil shape variables.

Table 4 shows the data for the TOGW and fuel burn optimizations, and Figure 13 compares the pressure contours, airfoil shapes, structural thicknesses, spanwise lift distributions, twist distributions, and $t / c$ distributions for these two 

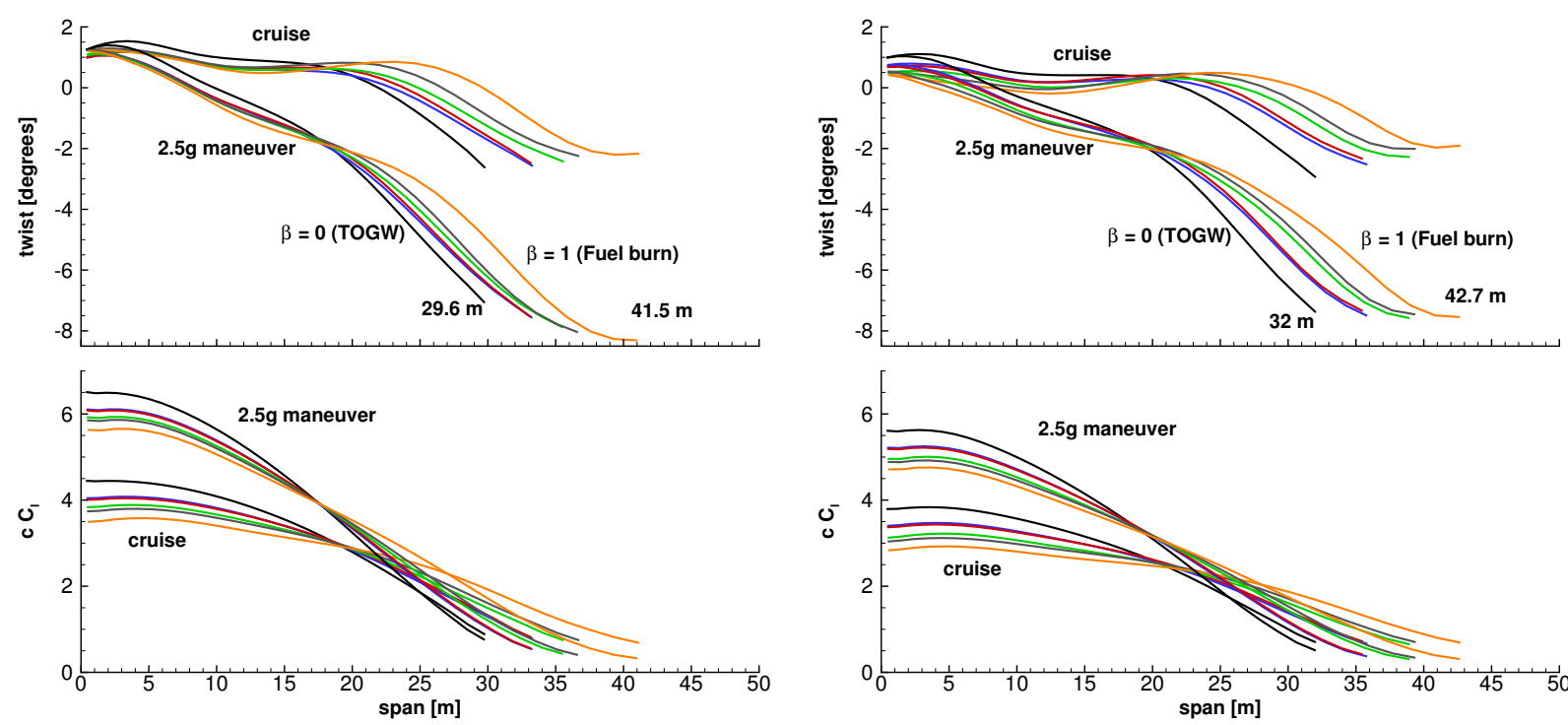

(a) Metallic

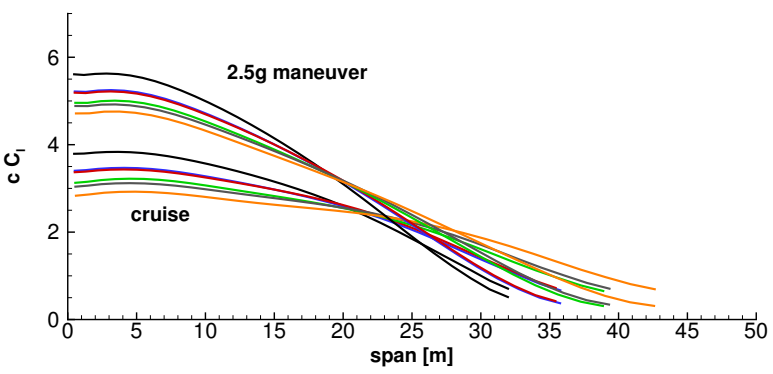

(b) Composite

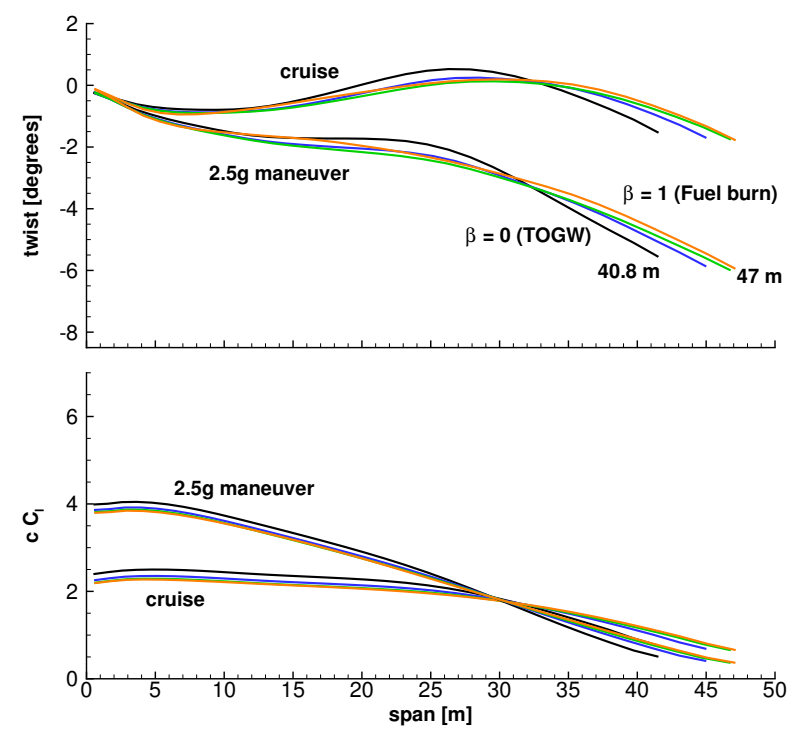

(c) CNT

Figure 10: Twist at cruise and the $2.5 \mathrm{~g}$ maneuver condition, as well as lift normalized by dynamic pressure at the cruise and $2.5 \mathrm{~g}$ maneuver conditions.

optimizations. ).

The performance of the baseline design was poor due to a large wave drag component and therefore is not used for comparison purposes. Both the fuel burn and TOGW were reduced for relative to the initial design in both optimizations.

As expected, the TOGW for the $\beta=0$ optimization is lower than the fuel burn optimization, but converse is not true: The fuel-burn optimized design has $0.7 \%$ high fuel burn. The reason for this discrepancy is that these optimizations were performed at a fixed altitude, and therefore the wing is not free to fly at the best point in its drag polar. For the fuel burn optimization, the final reference area was increased by $35 \%$ from the baseline value of $198.3 \mathrm{~m}^{2}$. With such a large increase in area, the fixed design altitude of $36000 \mathrm{ft}$ does not yield the best possible performance for the fuel burn optimized design. An altitude sweep was performed at constant lift for each of the two optimized designs. For the minimum TOGW design, the best altitude was $36,250 \mathrm{ft}$, which is close to the design altitude. However, the minimum fuel burn design, which has a significantly reduced wing loading, benefits from a 


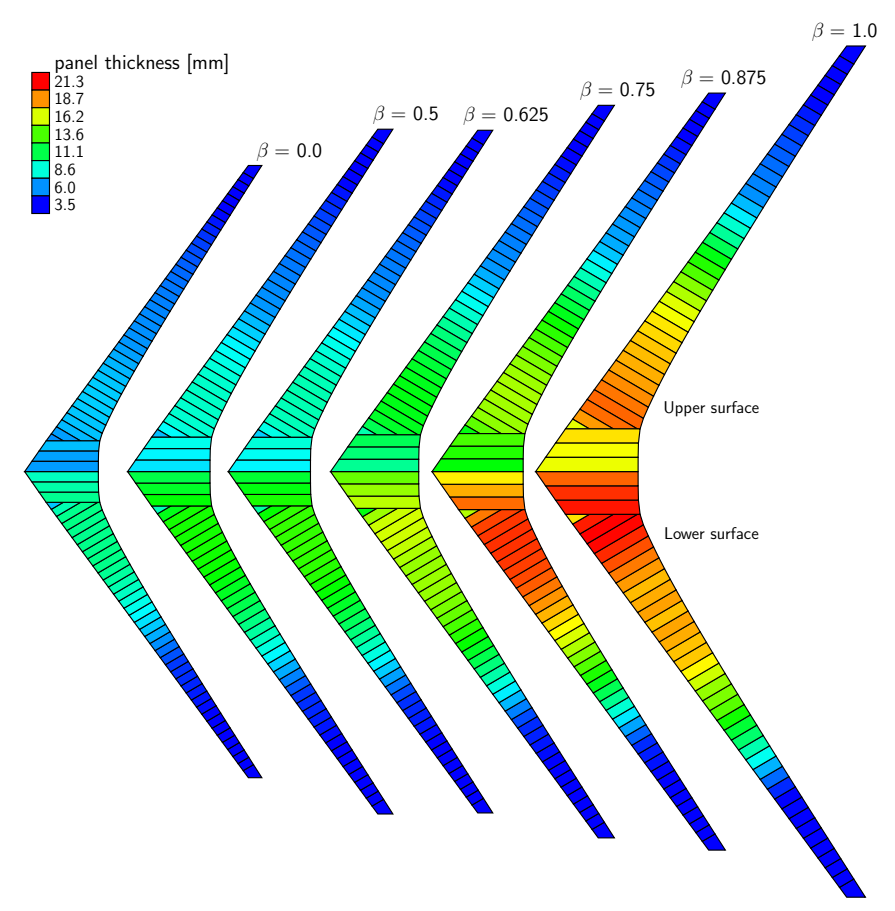

(a) Metallic

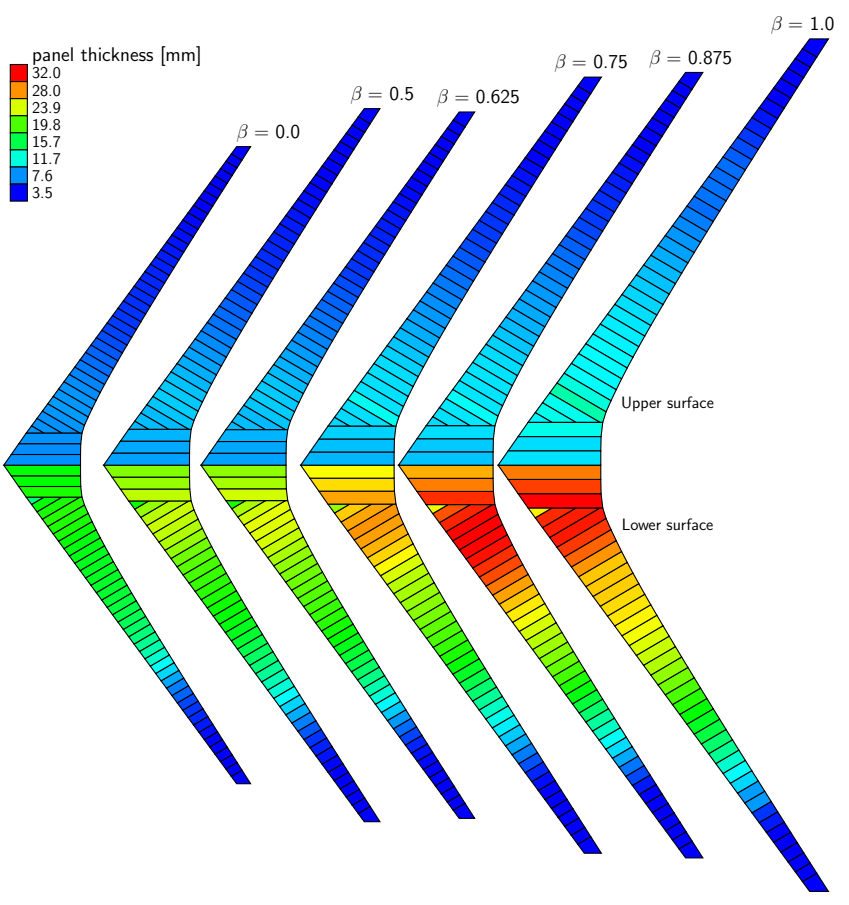

(b) Composite

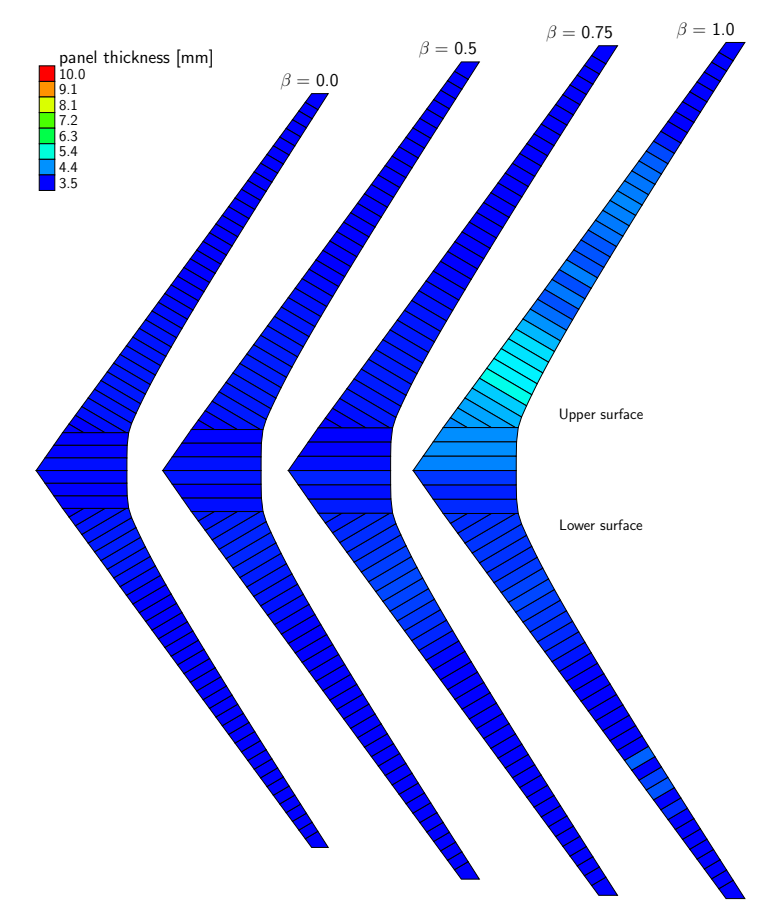

(c) $\mathrm{CNT}$

Figure 11: Skin thickness distributions for the conventional composite and CNT-based composite designs.

higher altitude, being the best at around $39000 \mathrm{ft}$. At this altitude, the fuel burn for the $\beta=1.0$ design is $71467 \mathrm{~kg}$, $3.7 \%$ lower than for the $\beta=0.0$. However, even correcting for the best altitude does not ensure that fuel burn design is optimal. This is because the optimization designed the airfoil cross sections for low wave drag at a much lower $C_{L}$ (for $36000 \mathrm{ft}$ ) than is experienced at $39000 \mathrm{ft}$ for the same lift. Thus, if large changes in wing area or mass are expected during the optimization, altitude variation, or a surrogate for altitude variation should be included as a design 


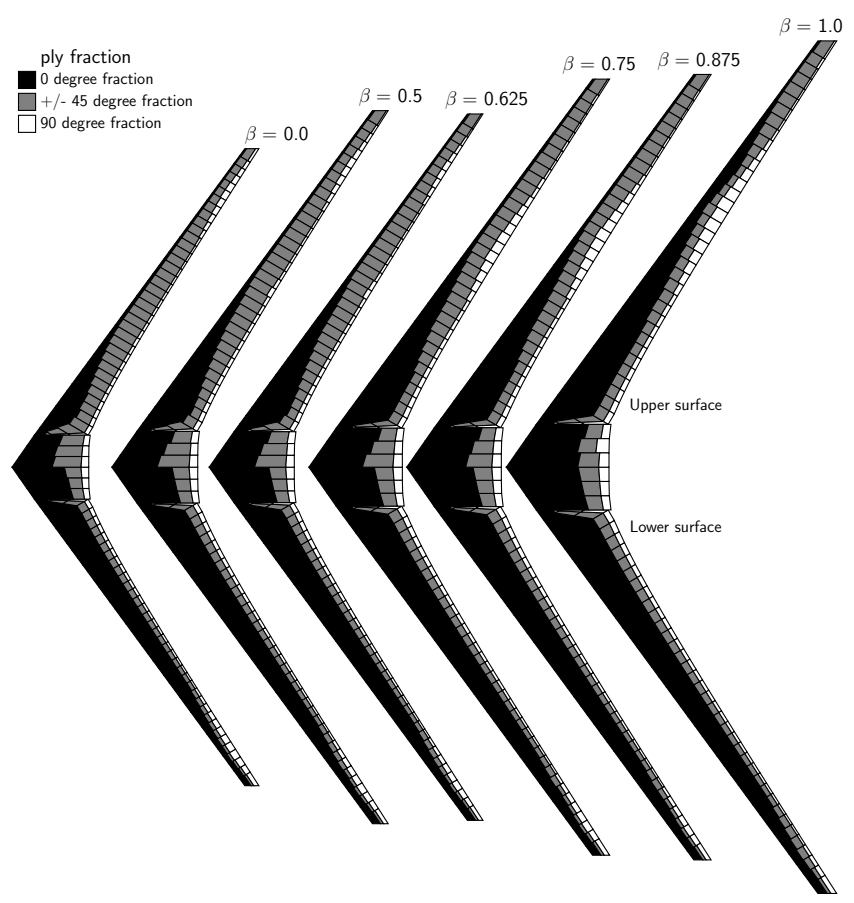

(a) Composite

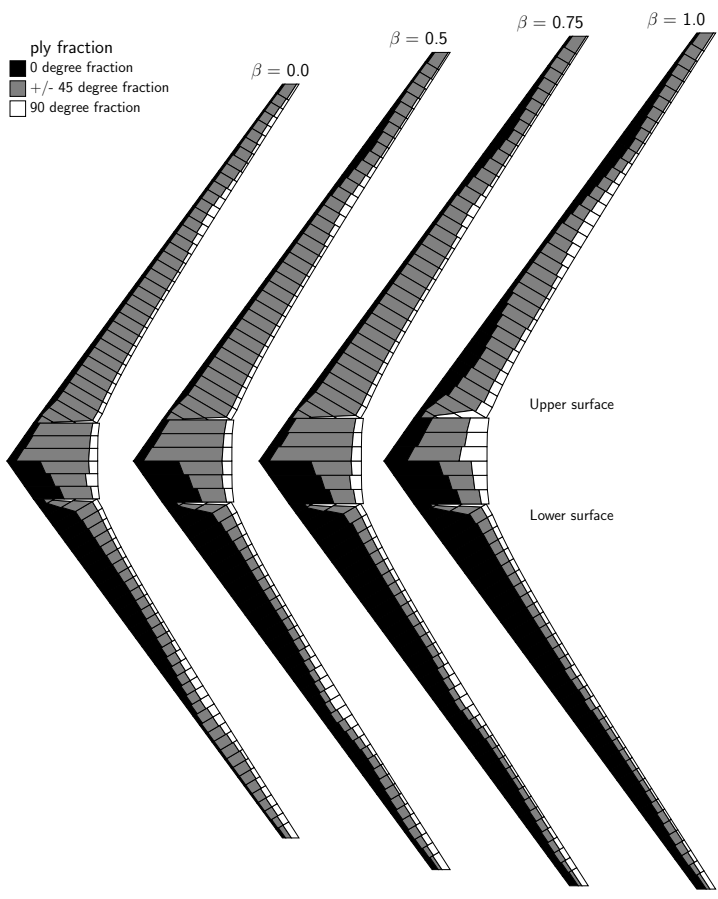

(b) CNT

Figure 12: Ply fractions for the conventional composite and CNT-based composite designs.

\begin{tabular}{llllllll}
\hline$\beta$ & TOGW $(\mathrm{kg})$ & Fuel Burn $(\mathrm{kg})$ & Wing Mass $(\mathrm{kg})$ & L/D & Span & Wing Area $\left(\mathrm{m}^{2}\right)$ & Aspect Ratio \\
\hline 0.0 & 268630 & 74242 & 26567 & 20.13 & 73.8 & 431.348 & 12.62 \\
1.0 & 290206 & 74754 & 47631 & 21.87 & 80.8 & 523.468 & 12.45 \\
\hline
\end{tabular}

Table 4: TOGW and fuel burn results for RANS-based aerostructural optimization

variable in the optimization problem.

The choice of objective function has a dramatic effect on nearly every aspect of the optimized design. The wing planform is the first major difference (see Figure 13). Both designs have roughly the same aspect ratio, but the fuel burn design wing span is $7 \mathrm{~m}$ longer and has a $21.4 \%$ larger wing area. Since th fuel burn objective places less emphasis on the empty weight, the wing span extends to lower the span-loading and thus lower the induced drag. This increase in span comes at a substantial penalty in terms of structural weight, $21064 \mathrm{~kg}$ or $79 \%$ of the TOGW optimized mass. This large wing mass is further explained by examining the distribution of thickness to chord ratio distributions. The average $t / c$ for the TOGW minimization is $33 \%$ higher than for the fuel burn design. However, the difference in the wingbox depth is slightly lower than this due to the larger chord of the minimum fuel burn wing. Further confirmation of the increased wingbox mass can be seen in the upper skin thickness distribution contours in Figure 13. With the exception of the more lightly loaded wing tips, the fuel burn design skin is thicker than the minimum TOGW design. These trends are consistent with previous aerostructural optimizations performed by the authors using Euler CFD [14].

The cruise lift distributions for both minimum fuel burn and minimum TOGW designs shown in Figure 13 are close to elliptical, but the TOGW design yield a closer match to this ideal distribution. However, there is a very large difference in the shape of the maneuver lift distributions. Both designs exhibit passive load alleviation-where the $2.5 \mathrm{~g}$ maneuver lift distribution is shifted inboard relative to the elliptical distribution-but this load alleviation is more pronounced for the minimum TOGW design.

For the fuel burn design, the corresponding twist distribution shows the additional passive aeroelastic wash-out that occurs at the maneuver condition. This additional downward twisting reduces the tip load, shifting the distribution inboard and lowering the bending moment on the structure. This behavior is consistent with the medium fidelity results. 


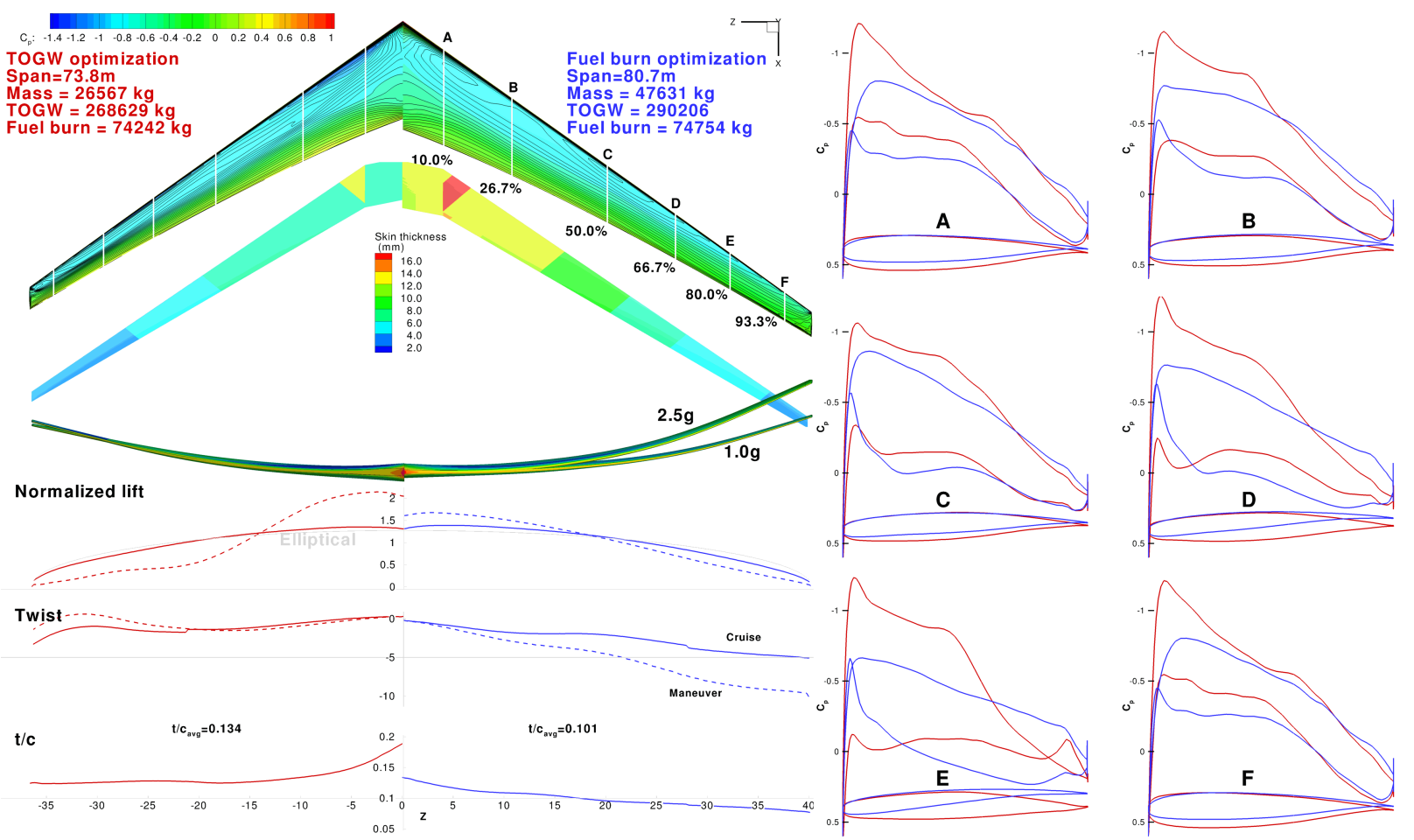

Figure 13: Comparison of TOGW and fuel burn optimized designs

The $2.5 \mathrm{~g}$ maneuver lift distribution for the TOGW design shows a completely opposite twist behavior: the wing twist actually decreases under the higher loading condition. Even so, the maneuver lift TOGW maneuver lift distribution has shifted further inboard and is even more favorable from a structural perspective. To explain this phenomenon, we examined the three-dimensional flow field of the TOGW $2.5 \mathrm{~g}$ maneuver condition (shown in Figure 14). The angle of attack for this simulation is $8.6^{\circ}$ ( $3^{\circ}$ larger than the $2.5 \mathrm{~g}$ fuel burn case), and a large portion of the wing has stalled, resulting in large region of separated flow. The gray-colored area in Figure 14 is the contour of -0.001 $x$-velocity, showing the region in which the flow direction has reversed. The slice at $66 \%$ semi-span shows the the $\tilde{\nu}_{\mathrm{SA}}$ variable, which indicates a region of very large eddy viscosity that is consistent with separated flow. For the fuel burn design, the flow remains attached and the eddy viscosity production is much, much lower. Although stall is not desirable within the flight envelope (especially tip stall), the optimizer exploited the fact that no stall constraint is imposed to implement an extremely effective way to alleviate the loads. While the simulation of the stalled wing did converge in the steady state sense, in reality this flow condition is unsteady and would be accompanied by buffeting. Further, such a flow condition would most likely significantly reduce the control effectiveness of any outboard control surface.

The "optimal" flow condition for the $2.5 \mathrm{~g}$ maneuver condition clearly demonstrates some of the unintended consequences of high fidelity aerostructural optimization significant design freedom. Without an explicit stall constraint, the optimizer sees this is a perfectly valid design, that is superior to the bend-twist coupling load alleviation. We suspect that this type of design may be an artifact of the single $2.5 \mathrm{~g}$ maneuver Mach number and lift coefficient. Other flow conditions, especially at lower lift coefficient will not be stalled and thus may violate the stress and buckling constraints.

\section{Conclusions}

In this paper we investigated the differences between optimal wings for three different materials: aluminum alloy, a conventional carbon composite and a hypothetical CNT-based composite. The objective was to understand the design trends for the various materials and to quantify how much the performance can be improved. In the particular case of the CNT composite, we assumed that both stiffness and strength were about one order of magnitude greater than conventional composites, which is well beyond what is currently possible. We quantified the potential benefits of the 


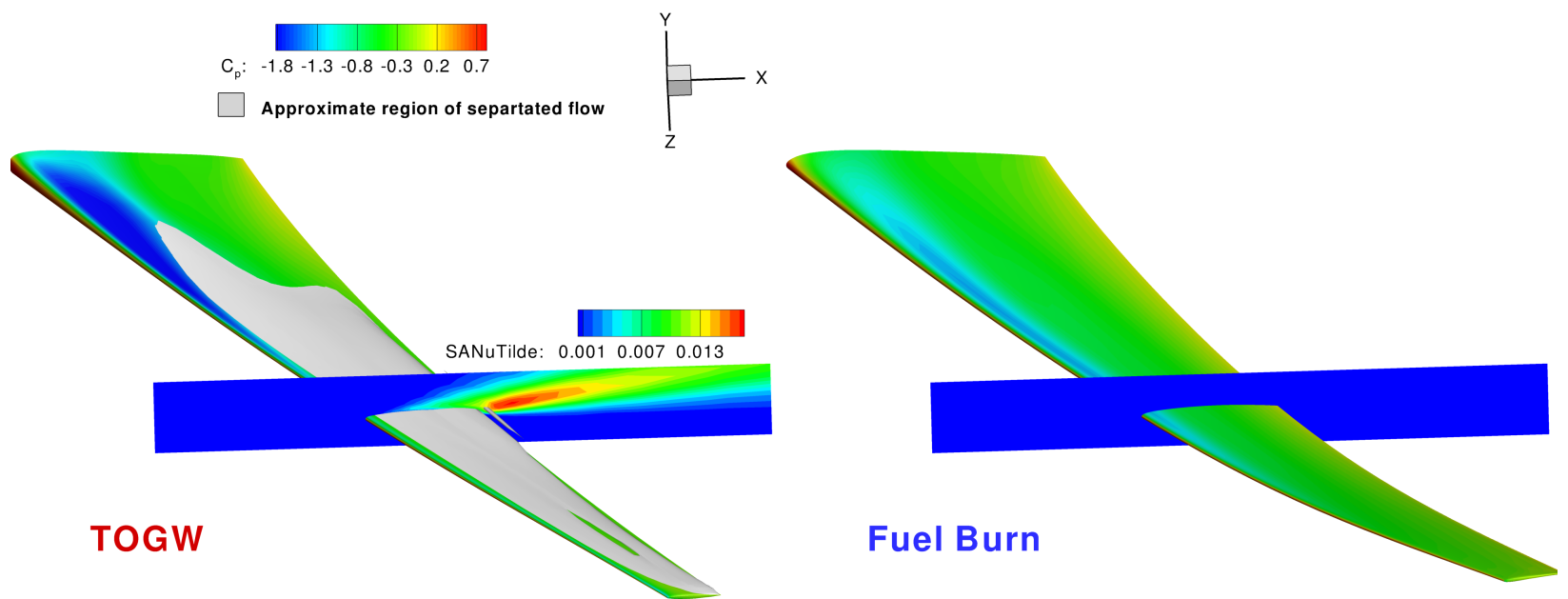

Figure 14: Flow visualization for the $2.5 \mathrm{~g}$ maneuver conditions.

various materials by obtaining Pareto fronts with respect to fuel burn and TOGW, since the real objective function in aircraft design is somewhere between these two objective functions, but depends on a number of factors such as fuel price.

The design optimization approach was to perform aerostructural optimizations whereby the structural sizing and the aerodynamic shape are optimized simultaneously. We showed that the Pareto front obtained through aerostructural optimization was significantly better than the sequential results with respect to both objectives. The designs obtained through aerostructural optimization exhibited spanwise lift distributions that were close to elliptical at the cruise flight conditions, while being able to take advantage of passive load alleviation at the critical structural load conditions. Thus, an aerostructural optimization approach is essential to obtain optimal static aeroelastic tailored wings and to evaluate the benefits of different materials.

The use of more advanced materials enabled reductions in both objectives, resulting in a movement of the Pareto front towards the origin. The minimum fuel burn composite wing reduced the fuel burn by $7.7 \%$ when compared to the corresponding metallic wing, and the minimum TOGW composite wing reduced the TOGW by $8.4 \%$. The corresponding gains for the CNT composite versus the conventional composite wing were more modest: 5.2 and $5.6 \%$, respectively. This seems to be mostly due to the minimum structural thickness constraint, which was active for a large portion of the CNT wing. Since we use the same minimum thickness as the conventional composite, this minimum value requires further investigation.

The optimal wing trends were consistent among the different materials: the minimum fuel burn wings were found to be longer, heavier, thinner, more flexible, and more lightly loaded than their minimum TOGW counterparts. The optimal composite wings exhibited larger spans than the metallic wings, and the CNT wings had even larger spans, reaching a maximum of almost $97 \mathrm{~m}$ for the minimum fuel burn case.

In addition to the aerostructural optimizations based on panel code aerodynamics, we also performed a few RANSbased aerostructural optimizations. While the trends shown by these higher fidelity results were similar, the fuel burn and TOGW values were quite different, and the resulting spans varied a lot less. These differences were in part due to an offset in the drag due to the physics considered, but we also found that some of the optimal wings were not flying at their optimal lift coefficient. To address this, we plan to include the cruise altitude as an additional design variable.

The RANS results also added an additional insight to this study. The passive load alleviation that we had observed so far consisted in exploiting the bend-twist coupling to twist down the outer wing at the critical load conditions to reduce the lift in that area, resulting in a reduction in bending moments. However, the RANS result for optimal TOGW discovered it could stall the outer wing to achieve even more dramatic load alleviation. This is obviously not a desirable flight condition and points out the need to enforce a stall constraint in our optimizations. 


\section{Acknowledgments}

Funding for this research was provided by NASA under grant number NNX11AI19A. The authors would like to thank Christine Jutte, Bret Stanford, and Karen Taminger for their help in defining this project and for their suggestions in the writing of this paper.

\section{References}

[1] S. Balay, W. D. Gropp, L. C. McInnes, and B. F. Smith. Efficient management of parallelism in object oriented numerical software libraries. In E. Arge, A. M. Bruaset, and H. P. Langtangen, editors, Modern Software Tools in Scientific Computing, pages 163-202. Birkhäuser Press, 1997.

[2] S. Brown. Displacement extrapolation for CFD+CSM aeroelastic analysis. In 38th Structures, Structural Dynamics, and Materials Conference, April 1997. doi:10.2514/6.1997-1090. AIAA97-1090.

[3] I. R. Chittick and J. R. R. A. Martins. An asymmetric suboptimization approach to aerostructural optimization. Optimization and Engineering, 10(1):133-152, Mar. 2009. doi:10.1007/s11081-008-9046-2.

[4] P. E. Gill, W. Murray, and M. A. Saunders. SNOPT: An SQP algorithm for large-scale constrained optimization. SIAM Review, 47(1):99-131, 2005. doi:10.1137/S0036144504446096.

[5] B. Grossman, R. T. Haftka, P.-J. Kao, D. M. Polen, and M. Rais-Rohani. Integrated aerodynamic-structural design of a transport wing. Journal of Aircraft, 27(12):1050-1056, 1990. doi:10.2514/3.45980.

[6] E. J. Hopkins. Charts for predicting turbulent skin friction from the van Driest method (II). Technical Report TN D-6945, NASA, October 1972.

[7] A. Jameson. Computational aerodynamics for aircraft design. Science, 245:361-371, 1989.

[8] R. M. Jones. Mechanics of Composite Materials. Technomic Publishing Co., 1996.

[9] C. V. Jutte, B. K. Stanford, C. D. Wieseman, and J. B. Moore. Aeroelastic tailoring of the NASA common research model via novel material and structural configurations. In Proceedings of the SciTech Conference, National Harbor, MD, January 2014.

[10] J. Katz and A. Plotkin. Low-Speed Aerodynamics. McGraw-Hill Inc., 1991.

[11] G. J. Kennedy and J. R. R. A. Martins. Parallel solution methods for aerostructural analysis and design optimization. In Proceedings of the 13th AIAA/ISSMO Multidisciplinary Analysis Optimization Conference, Fort Worth, TX, September 2010. AIAA 2010-9308.

[12] G. J. Kennedy and J. R. R. A. Martins. A comparison of metallic and composite aircraft wings using aerostructural design optimization. In 14th AIAA/ISSMO Multidisciplinary Analysis and Optimization Conference, Indianapolis, IN, Sep. 2012. AIAA-2012-5475.

[13] G. J. Kennedy and J. R. R. A. Martins. A laminate parametrization technique for discrete ply angle problems with manufacturing constraints. Structural and Multidisciplinary Optimization, 48(2):379-393, August 2013. doi:10.1007/s00158-0130906-9.

[14] G. K. W. Kenway and J. R. R. A. Martins. Multi-point high-fidelity aerostructural optimization of a transport aircraft configuration. Journal of Aircraft, 2013. doi:10.2514/1.C032150. (In press).

[15] G. K. W. Kenway, G. J. Kennedy, and J. R. R. A. Martins. A scalable parallel approach for high-fidelity steady-state aeroelastic analysis and adjoint derivative computations. AIAA Journal, 2012. (In press).

[16] I. Kroo. Aircraft design: Synthesis and analysis, November 2013. URL http://adg.stanford.edu/aa241/ AircraftDesign.html.

[17] I. M. Kroo. Drag due to lift: Concepts for prediction and reduction. Annual Review of Fluid Mechanics, 33:587-617, Nov. 2000.

[18] Z. Lyu and J. R. R. A. Martins. Aerodynamic design optimization studies of a blended-wing-body aircraft. Journal of Aircraft, 2014. (In press).

[19] Z. Lyu, G. K. Kenway, C. Paige, and J. R. R. A. Martins. Automatic differentiation adjoint of the Reynolds-averaged NavierStokes equations with a turbulence model. In 21st AIAA Computational Fluid Dynamics Conference, San Diego, CA, Jul 2013. doi:10.2514/6.2013-2581. 
[20] C. A. Mader and J. R. R. A. Martins. Stability-constrained aerodynamic shape optimization of flying wings. Journal of Aircraft, 50(5):1431-1449, September 2013. doi:10.2514/1.C031956.

[21] C. A. Mader, J. R. R. A. Martins, J. J. Alonso, and E. van der Weide. ADjoint: An approach for the rapid development of discrete adjoint solvers. AIAA Journal, 46(4):863-873, Apr. 2008. doi:10.2514/1.29123.

[22] B. Malone and W. Mason. Multidisciplinary optimization in aircraft design using analytic technology models. Journal of Aircraft, 32(2):431-438, mar-apr 1995. doi:10.2514/3.46734.

[23] J. R. R. A. Martins and J. T. Hwang. Review and unification of methods for computing derivatives of multidisciplinary computational models. AIAA Journal, 51(11):2582-2599, 2013. doi:10.2514/1.J052184.

[24] J. R. R. A. Martins and A. B. Lambe. Multidisciplinary design optimization: A survey of architectures. AIAA Journal, 51(9): 2049-2075, Sep 2013. doi:10.2514/1.J051895.

[25] J. R. R. A. Martins, J. J. Alonso, and J. J. Reuther. High-fidelity aerostructural design optimization of a supersonic business jet. Journal of Aircraft, 41(3):523-530, 2004. doi:10.2514/1.11478.

[26] J. R. R. A. Martins, J. J. Alonso, and J. J. Reuther. A coupled-adjoint sensitivity analysis method for high-fidelity aerostructural design. Optimization and Engineering, 6(1):33-62, Mar. 2005. doi:10.1023/B:OPTE.0000048536.47956.62.

[27] R. E. Perez, P. W. Jansen, and J. R. R. A. Martins. pyOpt: a Python-based object-oriented framework for nonlinear constrained optimization. Structural and Multidisciplinary Optimization, 45(1):101-118, January 2012. doi:10.1007/s00158-011-0666-3.

[28] N. M. K. Poon and J. R. R. A. Martins. An adaptive approach to constraint aggregation using adjoint sensitivity analysis. Structural and Multidisciplinary Optimization, 34(1):61-73, 2007. doi:10.1007/s00158-006-0061-7.

[29] Y. Saad and M. H. Schultz. GMRES: A generalized minimal residual algorithm for solving nonsymmetric linear systems. SIAM Journal on Scientific and Statistical Computing, 7(3):856-869, 1986. doi:10.1137/0907058.

[30] W. J. Stroud and N. Agranoff. Minimum mass design of filamentary composite panels under combined loads: Design procedure based on simplified buckling equations. Technical report, NASA Langley Research Center, Hampton, VA 23665, October 1976.

[31] E. van der Weide, G. Kalitzin, J. Schluter, and J. J. Alonso. Unsteady turbomachinery computations using massively parallel platforms. In Proceedings of the 44th AIAA Aerospace Sciences Meeting and Exhibit, Reno, NV, 2006. AIAA $2006-0421$.

[32] J. C. Vassberg. A unified baseline grid about the common research model wing-body for the fifth aiaa cfd drag prediction workshop. In 29th Applied Aerodynamics Conference, Honolulu, Hawaii, June 2011. doi:10.2514/6.2011-3508. AIAA 2011-3508.

[33] S. Venkatamaran and R. T. Haftka. Structural optimization complexity: what has Moore's law done for us? Structural and Multidisciplinary Optimization, 28:375-387, 2004.

[34] V. B. Venkayya. Structural optimization: A review and some recommendations. International Journal for Numerical Methods in Engineering, 13(2):203-228, 1978. doi:10.1002/nme.1620130202.

[35] S. Wakayama and I. Kroo. Subsonic wing planform design using multidisciplinary optimization. Journal of Aircraft, 32(4): 746-753, 1995. doi:10.2514/3.46786. 